\title{
Yang-Mills Fields on Cylindrical Manifolds and Holomorphic Bundles I
}

\author{
Guang-Yuan Guo \\ Department of Mathematics, Wells Hall, Michigan State University, East Lansing, MI 48824-1027, \\ USA. E-mail: gyguo@math.msu.edu
}

Received: 31 July 1995/Accepted: 14 February 1996

\begin{abstract}
We give complex holomorphic descriptions of Yang-Mills instantons on tubular four manifolds with nontrivial circle bundles over Riemann surfaces as section.
\end{abstract}

\section{Introduction}

This is the first of two installments in which we study Yang-Mills instantons, i.e. anti-self-dual connections with finite Yang-Mills energy on tubular manifolds $Y \times R$, where $Y$ is a non-trivial circle bundle over a Riemann surface.

For a circle bundle $Y$ on a Riemann surface $\Sigma$, the space $Y \times R$ admits a complex structure and can be compactified to a ruled surface $S$ by, roughly speaking, adding a copy of $\Sigma$ to each end of the tube $Y \times R$. On the other hand, the finite energy condition and Uhlenbeck's compactness theorem implies that on a manifold with ends any instanton is asymptotic over each end to a flat connection on the section over the end. For a circle bundle $Y$ on a Riemann surface $\Sigma$, flat connections on $Y$ can be divided roughly into two classes, those with non-trivial holonomy along the fibre circle as one and those with trivial one as the other one. Accordingly instantons on $Y \times R$ can be divided into three classes, those with flat limits without holonomy along the fibre circle as the first one, those with flat limits with holonomy along the fibre circle as the second one and those with mixed limits as the last one. Roughly speaking, in this first installment, we prove that there is a natural injection of the set of instantons in the first class into the space of holomorphic bundles over $S$ which are flat along the two added divisors. In the next installment, we shall prove that there is a similar injective map from the set of instantons in the second class to the space of certain equivariant holomorphic bundles on a canonical covering of $S$, branched over the two added divisors, with some preferred filtration and unitary structures in the restrictions over the two divisors. Complex holomorphic description for instantons in the third class can be obtained as a corollary to these two results. For precise statements, see the main text.

To establish our main results, we need to show that given an appropriate instanton, there is naturally an associated holomorphic bundle with required properties 
and vice versa. We first go from holomorphic bundles to anti-self-dual connections. We use the evolution equation

$$
H^{-t}(s) \frac{\partial H^{t}(s)}{\partial s}=-i \Lambda F(s)
$$

(where $H^{t}$ denotes the transpose for $H$ and similarly for others in the article) to demonstrate the existence of an anti-self-dual connection with appropriate properties on $Y \times R$ from the holomorphic data provided. We then solve a " $\bar{\partial}$ " equation to show that the holomorphic bundle on $Y \times R$ determined by an instanton on $Y \times R$ extends to give a holomorphic bundle on the associated ruled surface $S$ with the corresponding structures.

The evolution equation method was first developed by Donaldson [6] in the context of holomorphic bundles over compact projective surfaces and was later generalized by Bando [2] and Simpson [19] for bundles over noncompact Kaehler manifolds. The method depends nontrivially on the geometry of the base manifold, and since the geometry of our base manifold $Y \times R$ is a combination of those considered by Bando [2] and Simpson [19], our argument is a combination of those used by Bando [2] and Simpson [19]. Since the tube metric used to deform the evolution equation is only a Hermitian metric with respect to the complex structure on $Y \times R$, we no longer have the convenience of the Kaehler geometry: we have to generalize some of the basic facts about the solutions of the evolution equation in the case of Kaehler base manifolds to the more general case of Hermitian base manifolds. This is, however, compensated by the fact that the spectral geometry of $Y \times R$ with the tube metric is relatively simple. In fact, the tube metric is conformal to a Kaehler metric, as we shall see in Sect. 3, and we could have used the Kaehler metric to deform the evolution equation as well, but we are unable to establish a couple of key properties of the heat kernel of $Y \times R$ with this metric.

The complex holomorphic approach to the study of instantons is of course not a new one. On the contrary, until recently, most of the applications of Donaldson's new invariants are achieved through algebraic holomorphic geometry. In complex dimension two, conformal geometry, which is the relevant geometry for the Yang-Mills instanton equation, interwines closely with Kaehler geometry, and this leads to the complex holomorphic description of the instanton moduli spaces on complex surfaces. Algebraic geometry is then applied to study these moduli spaces.

There are some new aspects to the problem in our situation, due to the fact that tubular manifolds are non-compact. First, in the compact case, holomorphic data used to describe the instanton moduli spaces is obtained more or less as a consequence of the Newlander-Nirenberg integrability theorem. In our case here, the integrability theorem only gives holomorphic data on a non-compact space, thus to obtain the relevant holomorphic data, we have to go a step further. Second, the analysis involved has to be on non-compact spaces. Technically it is mainly with these two new aspects of the problem that this paper is concerned.

As with the case of compact manifolds, the results in the paper can be viewed from a totally complex holomorphic point of view. In complex holomorphic geometry, one is interested in the existence of various Hermitian Yang-Mills connections on holomorphic bundles. As is now well known, on compact Kaehler manifolds the condition for the existence of such connections is that the relevant holomorphic 
bundle be stable, in the sense of Mumford. In another word, there is a one to one correspondence between the space of stable holomorphic bundles and the space of (irreducible) Hermitian Yang-Mills connections [18, 6, 22]. This is the KobayashiHitchin correspondence. For bundles on non-compact base manifolds, similar results are obtained by Bando [2], and Simpson [19], for rather different non-compact base manifolds. In complex dimension two, the notion of Hermitian Yang-Mills connection and instanton coincide. The results here thus can be viewed as another kind of Kobayashi-Hitchin correspondence. The basic difference is that the stability condition is replaced, in the case of bundles on manifolds with an infinite volume, by a condition which essentially is a boundary condition at infinity.

The rest of the paper is organised as follows: In Sect. 1, we describe the space of flat connections on a nontrivial circle bundle and collect some fundamental analytic results about asymptotic behaviour of instantons on tubular manifolds. These preliminary results are needed for the precise statments of our main results as well as their proof. In Sect. 2, after introducing some notation, we give a formal statement of our main results. In Sect. 3, we study the Kaehler and Hermitian structures on our cylinder $Y \times R$. This enables us to obtain in Sect. 4 some interesting properties of the heat kernel of the relevant linear operator. The results in Sects. 3 and 4 may be of independent interest. With the preparation in Sects. 3 and 4, we prove one of the main theorems in Sect. 5. We then complete the proof of the other main theorem in Sect. 6.

\section{Some Preliminary Results}

In this section we briefly discuss the space of flat connections on a non-trivial circle bundle $Y$ and collect some basic facts about the asymptotic behaviour of instantons on manifolds with tubular ends. These will be needed for the precise statements of our main results as well as their proof.

Let $M$ be a 4-manifold with a finite number of ends and $Y$ be a three manifold as in the last section. Let $A$ be an anti-self-dual connection on $P=M \times S U(2)$ with finite energy, i.e. $\int_{M}\left|F_{A}\right|^{2}<\infty$. By restricting $A$ to the slices $Y \times\{t\}$ over the end $Y \times R^{+}$, we obtain a family of connections $A(t)$ on $Y$. Generally $A(t)$ depends on the choice of the gauge of $P$ on $M$ over the end. Denote by $[A(t)]$ the image of $A(t)$ in the space $\mathcal{B}$ of equivalence classes of connections on $Y$, then $[A(t)]$ is welldefined. The following fundamental theorems concerning the asymptotic behavior of $[A(t)]$ are standard (see $[8,20])$.

Theorem A. The connection $[A(t)]$ converges in $C^{k}$ to a flat connection $\left[A_{\infty}\right]$ on $Y$ as $t \rightarrow \infty$.

Theorem B. If the limiting flat connection $\left[A_{\infty}\right]$ on $Y$ is a nondegenerate critical point of the Chern-Simons function on $\mathcal{B}$ in the sense of Bott, then there is a number $\delta>0$ such that

$$
\left|F_{A}\right| \leqq C e^{-\delta t}
$$

Morever, we can choose a gauge on the end $Y \times R^{+}$such that

$$
\left|\nabla_{A_{\infty}}^{k}\left(A-A_{\infty}\right)\right| \leqq C e^{-\delta t}, \quad k=0,1, \ldots
$$


Here $A_{\infty}$ denote the pull-back connection on the end $Y \times R^{+}$of some representative of $\left[A_{\infty}\right]$ by the obvious projection from $Y \times R^{+} \times S U(2)$ to $Y \times S U(2)$ given by the chosen gauge.

A critical point of a function on a space is said to be nondegenerate in the sense of Bott if the Hessian of the function is nondegenerate on the subspace normal to the critical set at the point. Such critical points are sometimes called Morse-Bott points.

By Theorem $\mathrm{A}$, the moduli space $\mathcal{M}$ of finite energy instantons on $M$ is divided into a number of subsets parametrized by the set of combinations of the same number as that of the ends of components of the space of $S U(2)$ flat connections on $Y$. In particular, when $M$ is a tube $Y \times R, \mathcal{M}$ is the disjoint union of its subsets $\mathcal{M}\left(\mathcal{F}_{-\infty}, \mathcal{F}_{\infty}\right)$, where $\mathcal{F}_{-\infty}$ and $\mathcal{F}_{\infty}$ run over all components of the space of flat $S U(2)$ connections on $Y$ and $\mathcal{M}\left(\mathcal{F}_{-\infty}, \mathcal{F}_{\infty}\right)$ is the subset of $\mathcal{M}$ consisting of instantons with limits in $\mathcal{F}_{-\infty}$ and in $\mathcal{F}_{\infty}$ over the two ends respectively.

We now limit ourself to the case $M=Y \times R$, where $Y$ is the unit circle bundle of a line bundle $L$ with nonzero Chern class over some Riemann surface $\Sigma$ of genus $g$. The fundamental group $\pi_{1}(Y)$ of $Y$ is a group generated by $2 g+1$ generators $\left\{a_{i}, b_{i}, c, i=1, \ldots, g\right\}$, where $a_{i}, b_{i}$ are the usual generators of $\pi_{1}(\Sigma)$ and $c$ is the generator in the fibre, subject to the following relations:

(i) $c$ is central;

(ii) $\prod_{i=1, \ldots, g}\left[a_{i}, b_{i}\right]=c^{c_{1}(L)}$.

Denote $\left|c_{1}(L)\right|$ by $\kappa . \kappa$ is a positive integer. One sees easily that the set of equivalence classes of flat connections on $Y$ consists of the components described as follows:

(a) $\mathcal{F}_{+}=\left\{\rho \in \operatorname{Hom}\left(\pi_{1}(Y), S U(2)\right) \mid \rho(c)=1\right\} /$ conjugation;

(b) $\mathcal{F}_{-}=\left\{\rho \in \operatorname{Hom}\left(\pi_{1}(Y), S U(2)\right) \mid \rho(c)=-1\right\} /$ conjugation;

(c) $\mathcal{F}_{j}=\left\{\rho \in \operatorname{Hom}\left(\pi_{1}(Y), S U(2)\right) \mid \rho(c)=\left(\begin{array}{ll}e^{\frac{j 2 \pi i}{\kappa}} & 0 \\ 0 & e^{-\frac{j 2 \pi u}{\kappa}}\end{array}\right)\right\} /$ conjugation, for $1 \leqq j<\frac{\kappa}{2}$.

See also [20].

It is easy to see that each of the components above are path connected and $\mathcal{F}_{+}$is identical to the space of flat $S U(2)$ connections on $\Sigma$. Clearly all the irreducible flat connections are contained in $\mathcal{F}_{+} \cup \mathcal{F}_{-}$. If $c_{1}(L)$ is odd, then all the flat connections contained in $\mathcal{F}_{-}$are irreducible. In particular, the flat connections contained in $\mathcal{F}_{j}$ are all reducible.

By the above discussion, the space $\mathcal{M}$ of instantons on $Y \times R$ is thus the disjoint union of its subsets as follows:

$$
\begin{aligned}
\mathcal{M}= & \mathcal{M}\left(\mathcal{F}_{+}, \mathcal{F}_{+}\right) \cup \mathcal{M}\left(\mathcal{F}_{-}, \mathcal{F}_{-}\right) \cup \cup_{i, j} \mathcal{M}\left(\mathcal{F}_{i}, \mathcal{F}_{j}\right) \\
& \cup \mathcal{M}\left(\mathcal{F}_{+}, \mathcal{F}_{-}\right) \cup \mathcal{M}\left(\mathcal{F}_{-}, \mathcal{F}_{+}\right) \cup \cup_{j} \mathcal{M}\left(\mathcal{F}_{+}, \mathcal{F}_{j}\right) \\
& \cup \cup_{i} \mathcal{M}\left(\mathcal{F}_{i}, \mathcal{F}_{+}\right) \cup \cup_{j} \mathcal{M}\left(\mathcal{F}_{-}, \mathcal{F}_{j}\right) \cup \cup_{i} \mathcal{M}\left(\mathcal{F}_{i}, \mathcal{F}_{-}\right) .
\end{aligned}
$$

Let $\mathcal{F}_{+}^{*}$ now be the smooth part of $\mathcal{F}_{+}$and let $\rho \in \mathcal{F}_{+}^{*}$. The tangent space of $\mathcal{B}^{*} \quad$ at $\quad \rho$ is $T_{\rho} \mathcal{B}^{*}=\left\{a \in \Omega^{1}(a d P) \mid \tilde{d}_{\rho}^{*} a=0\right\} \quad$ and that of $\mathcal{F}^{*}$ is $T_{\rho} \mathcal{F}^{*}$ $=\left\{a \in \Omega^{1}(a d P) \mid\left(\tilde{d}_{\rho}+\tilde{d}_{\rho}^{*}\right) a=0\right\}$. Thus the subspace of $T_{\rho} \mathcal{B}^{*}$ normal to $\mathcal{F}^{*}$ is 
$N_{\rho} \mathcal{F}^{*}=\left\{a \in \Omega^{1}(a d P) \mid a \in \operatorname{Im}\left(\tilde{d}_{\rho}^{*}\right)\right\}$. On the other hand, the Hessian $H_{\rho}$ of the Chern-Simons function at $\rho$ is given by

$$
H_{\rho}(a, b) \mapsto-\int_{Y} \operatorname{Tr}\left(\tilde{d}_{\rho} a \wedge b\right)=\left\langle * \tilde{d}_{\rho} a, b\right\rangle
$$

We see that the Hessian $H_{\rho}$ is nondegenerate in $N_{\rho} \mathcal{F}^{*}$, i.e. $\rho \in \mathcal{F}^{*}$ is a Morse-Bott point.

Corollary a). Let $A$ be a finite energy instanton on $Y \times R$. If $\left[A_{ \pm \infty}\right] \in \mathcal{F}^{*}$, then there exist numbers $\delta>0, C>0$ such that

$$
\left|F_{A}\right| \leqq C \cdot e^{-\delta|t|}
$$

Moreover, we can choose a gauge on $Y \times R$ such that

$$
\left|\nabla_{A_{ \pm \infty}}^{k}\left(A-A_{ \pm \infty}\right)\right| \leqq C \cdot e^{-\delta|t|}, \quad k=0,1, \ldots
$$

Here $A_{ \pm \infty}$ denote the pull-back connection on the end $Y \times( \pm T, \pm \infty)$, for $T>0$ large enough, of some representative of $\left[A_{ \pm \infty}\right]$ on $Y$ by the obvious projection of $Y \times R \times S U(2)$ to $Y \times S U(2)$ given by the chosen gauge.

Similarly since $\mathcal{F}_{j}$ are smooth path components of the space of flat connections on $Y$, every point in these components is a Morse-Bott point. Therefore

Corollary b). The result in Corollary a) still holds if $\left[A_{ \pm \infty}\right] \in \mathcal{F}_{j}$.

In fact, according to Taubes [20], a stronger result holds. Taubes result says that if $Y$ is a circle bundle with negative Chern class, then the curvature of any instanton on $M$ decays exponentially over the end $Y \times R^{+}$. It follows trivially from this that if $Y$ is a circle bundle with positive Chern class, then the curvature of any instanton on $Y \times R$ decays exponentially over the end $Y \times(-\infty, 0]$.

Given any flat connection $A_{\infty}$ with $\left[A_{\infty}\right] \in \mathcal{F}_{j}$, we can associate with it a holomorphic flat line bundle $L_{A_{\infty}}$ on $\Sigma$ in a canonical way. Indeed the representation of $\pi_{1}(Y)$ corresponding to $A_{\infty}$ splits into two abelian representations in the eigenspaces of the central element $c$, with corresponding eigenvalues $\frac{j}{\kappa}$ and $-\frac{j}{\kappa}$. Each factor representation gives rise in an obvious way to an abelian representation of $\Pi_{1}(\Sigma)$, hence determines a flat line bundle on $\Sigma$. Define the flat line bundle $L_{\infty}$ on $\Sigma$ associated with $A_{\infty}$ to be the one correponding to the eigenvalue $\frac{j}{\kappa}$. The flat structure also determines a holomorphic structure on $L_{\infty}$.

\section{Statements of the Main Theorems}

Let $\left(E, H_{0}\right)$ be a fixed complex Hermitian bundle over the tube $Y \times R$, for a three manifold $Y$, associated with the principal $S U(2)$ bundle $Y \times R \times S U(2)$ on $Y \times R$. We identify connections on $Y \times R \times S U(2)$ with metric connections on $E$. Define $\mathcal{M}$ to be the space of equivalence classes of anti-self-dual connections with finite energy, i.e., instantons on $\left(E, H_{0}\right)$, the equivalence relation being the usual unitary gauge equivalence between connections on $\left(E, H_{0}\right)$. By the discussion in Sect. 1, we know that for a circle bundle $Y$ on a Riemann surface $\Sigma$ with non-zero first Chern class, the space $\mathcal{M}$ is the disjoint union of its subsets parametrized by the 
set of pairs of the path components of the space $\mathcal{F}$ of the space of flat connections on $Y$.

On the other hand, for a circle bundle $Y$ there are natural complex holomorphic data associated with $Y \times R . Y \times R$ can be identified as the complement to the zero section of a holomorphic line bundle $L$ on $\Sigma$ with first Chern class $c_{1}(L)=c_{1}(Y)$, and has a natural complex compactification $S$ which is the holomorphic $C P^{1}$ bundle associated with $L$. Clearly $S \backslash Y \times R$ is the union of the zero section and the section of points at "infinity" each of which is a copy of $\Sigma$. Denote the zero section by $\Sigma_{0}$ and the section of points at "infinity" by $\Sigma_{\infty}$. We now consider holomorphic bundles on $S$ with certain structures.

Let $\mathcal{E}_{c}(+,+)$ be the set of rank two holomorphic bundles on $S$ with trivial first Chern class such that their restrictions over $\Sigma_{0}$ and $\Sigma_{\infty}$ are unitary flat with some given preferred unitary structures. There are a few slightly different kinds of symmetries in $\mathcal{E}_{c}(+,+)$. First we forget the unitary structures over $\Sigma_{0}$ and $\Sigma_{\infty}$ and consider the ordinary equivalence relation of holomorphic bundle isomorphism in $\mathcal{E}_{c}(+,+)$. We define $\mathcal{M}_{c}(+,+)$ to be the resulting space of equivalence classes of elements of $\mathcal{E}_{c}(+,+)$ under this equivalence relation. Secondly, we also need to consider holomorphic bundle isomorphisms which are unitary with respect to the given unitary structures over the end $\Sigma_{0}$ or $\Sigma_{\infty}$ depending on the sign of the Chern class of $Y$. If the sign of the Chern class of $Y$ is positive, we define two bundles in $\mathcal{E}_{c}(+,+)$ to be equivalent if they are holomorphically isomorphic by an isomorphism which respects the given preferred unitary structures of the two bundles over the end $\Sigma_{0}$, and define $\mathcal{M}_{u c}(+,+)$ to be the resulting space of equivalence classes of elements of $\mathcal{E}_{c}(+,+)$. Similarly, if the sign of the Chern class of $Y$ is negative, we define the space $\mathcal{M}_{c u}(+,+)$. We could of course also consider holomorphic bundle isomorphisms which are unitary with respect to the given unitary structures over both ends and define the resulting moduli space $\mathcal{M}_{u}(+,+)$. It turns out, however, the relevant spaces in our descriptions of instanton moduli space $\mathcal{M}^{*}\left(\mathcal{F}_{+}, \mathcal{F}_{+}\right)$are the preceding ones rather than this $\mathcal{M}_{u}(+,+)$. This is perhaps not surprising as the topology of $Y$ should enter in our picture somewhere.

In general, the spaces $\mathcal{M}_{c}(+,+)$ and $\mathcal{M}_{c u}(+,+)$ or $\mathcal{M}_{u c}(+,+)$ are different. There is an obvious projection map from $\mathcal{M}_{u c}(+,+)$ or $\mathcal{M}_{c u}(+,+)$ to $\mathcal{M}_{c}(+,+)$ by forgetting the unitary structures. Let $\mathcal{M}_{c}^{*}(+,+)$ be the part of $\mathcal{M}_{c}(+,+)$ consisting of equivalence classes of bundles in $\mathcal{E}_{c}(+,+)$ whose flat structures over $\Sigma_{0}$ and $\Sigma_{\infty}$ are irreducible and similarly $\mathcal{M}_{u c}^{*}(+,+)$ or $\mathcal{M}_{c u}^{*}(+,+)$, then it is easy to see that the projection is an 1-1 map from $\mathcal{M}_{c u}^{*}(+,+)$ or $\mathcal{M}_{u c}^{*}(+,+)$ onto $\mathcal{M}_{c}^{*}(+,+)$. The map is obviously onto. Now on an irreducible holomorphic bundle, flat unitary structures are unique up to a constant, thus if $\mathcal{E}_{1}$ and $\mathcal{E}_{2}$ are two holomorphic bundles representing the same element of $\mathcal{M}_{c}^{*}(+,+)$, i.e. they are isomorphic by a holomorphic bundle isomorphism, $h$ say, then the flat unitary structures of $\mathcal{E}_{1}$ and $\mathcal{E}_{2}$ over the end $\Sigma_{0}$ or $\Sigma_{\infty}$, depending on the sign of Chern class of $Y$, differ by a constant under $h$. Multiplying $h$ with this constant gives a holomorphic bundle isomorphism between $\mathcal{E}_{1}$ and $\mathcal{E}_{2}$ which is unitary with respect to the given unitary structures of the two bundles over the specific end. Thus $\mathcal{E}_{1}$ and $\mathcal{E}_{2}$ represent the same element of $\mathcal{M}_{u c}^{*}(+,+)$ or $\mathcal{M}_{c u}^{*}(+,+)$.

Before we state the main results of this article, we simplify some of the notation introduced in the last chapter. We shall replace $\mathcal{M}\left(\mathcal{F}_{+}, \mathcal{F}_{+}\right)$by $\mathcal{M}(+,+)$ and shall simplify the notation for other subsets of $\mathcal{M}$ in a similar way. Thus, for example, $\mathcal{M}^{*}(+,+)$ will be the subset of $\mathcal{M}(+,+)$ consisting of instantons with irreducible 
flat limits in $\mathcal{F}_{+}$. Also let $\Omega_{0}$ be the form associated with the Hermitian tube metric on $Y \times R$ introduced in Sect. 3 (see the discussion before Lemma 3.2).

The main theorems of this article can now be stated as follows.

Theorem 2.1. Let $\mathcal{E} \in \mathcal{E}_{c}(+,+)$ be a holomorphic bundle over $S$ representing an element of $\mathcal{M}_{c}(+,+)$. There exists a unique Hermitian metric $H$ on $\left.\mathcal{E}\right|_{Y \times R}$ for which the following statements hold:

(1) The curvature $F_{H}$ of the Chern connection $A_{H}$ of $\left.(\mathcal{E}, H)\right|_{Y \times R}$ satisfies

$$
i \Lambda F_{H}=0 \text {, }
$$

where $\Lambda$ is the adjoint to the wedge product on forms by $\Omega_{0}$ on $Y \times R$.

(2) $H$ is bounded in the sense that for any smooth Hermitian metric $K$, say, on $\mathcal{E}$, there is a positive number $C$ such that $d(H, K)<C$, where $d$ is the distance in the space of Hermitian metrics (see [6, 16]). Moreover, if $c_{1}(Y)>0, H$ extends continuously over $\Sigma_{0}$ and coincides with the given unitary structure of $\mathcal{E}$ over $\Sigma_{0}$, and if $c_{1}(Y)<0, H$ extends over $\Sigma_{\infty}$ and coincides with the given unitary structure of $\mathcal{E}$ over $\Sigma_{\infty}$.

(3) If $c_{1}(Y)>0$, then the connection $A_{H}$ on $Y \times R$ has a flat limit $\left[A_{-\infty}\right]$ over the end $Y \times\{-\infty, 0]$ which is given by the lift to $Y$ of the given flat unitary structure of $\mathcal{E}$ over $\Sigma_{0}$. If $c_{1}(Y)<0$, then the connection $A_{H}$ has a flat limit $\left[A_{\infty}\right]$ over the end $Y \times[0, \infty\}$ which is given by the lift to $Y$ of the given flat unitary structure of $\mathcal{E}$ over $\Sigma_{\infty}$.

Theorem 2.2. There is an injective map from $\mathcal{M}^{*}(+,+)$ to $\mathcal{M}_{c}^{*}(+,+)$.

Remark. Our original goal is to prove that the above map is a one to one correspondence between $\mathcal{M}^{*}(+,+)$ and $\mathcal{M}_{c}^{*}(+,+)$. Theorem 2.1 gives a map from the set $\mathcal{M}_{c}^{*}(+,+)$ to the set of gauge equivalence classes of ASD connections on $Y \times R$, and in a suitable sense the map is a left inverse to the map of Theorem 2.2. But this still falls short of what we would like to prove: the map is actually the inverse to the map in Theorem 2.2. We are unable to prove that for a general class of such bundles, the corresponding gauge equivalence class of ASD connections on $Y \times R$ have finite Yang-Mills energy. For the very same reason, we have to state our results in a less strong way than we would like to when we give complex descriptions of the sets $\mathcal{M}\left(F_{i}, F_{j}\right)$ in part II.

The map in Theorem 2.2 would also be onto if the following conjectural statements hold for the metric $H$ in Theorem 2.1, in addition to (1), (2), and (3).

$\left(2^{\prime}\right)$ The Hermitian metric $H$ extends not only over one end as in (2), but over both the ends $\Sigma_{0}$ and $\Sigma_{\infty}$. Moreover, the restrictions of $H$ to the two ends are constant under the flat gauges of $\mathcal{E}$.

$\left(3^{\prime}\right)$ The connection $A_{H}$ is an instanton with

$$
\left\|F_{H}\right\|_{L^{2}}^{2}=8 \pi^{2} c_{2}(\mathcal{E}) \text {, }
$$

where $c_{2}(\mathcal{E})$ denotes the second Chern number of the bundle $\mathcal{E}$. The flat limits $\left[A_{-\infty}\right]$ and $\left[A_{\infty}\right]$ of the instanton $A_{H}$ coincide with the lifts to $Y$ of the given unitary flat structures $\mathcal{E}$ over $\Sigma_{0}$ and $\Sigma_{\infty}$ respectively.

As a consequence of the discussion in the appendix, we shall see that $\left(2^{\prime}\right)$ implies $\left(3^{\prime}\right)$. Thus if $\left(2^{\prime}\right)$ is true, then the conjectural statement below would also be true.

There is a 1-1 correspondence between $\mathcal{M}^{*}(+,+)$ and $\mathcal{M}_{c}^{*}(+,+)$. 


\section{Hermitian and Kaehler Structures on $Y \times R$}

Let $Y \stackrel{\pi}{\longrightarrow} \Sigma$ be a circle bundle over a Riemann surface $\Sigma$. We identify $Y$ with the unit circle bundle of some Hermitian line bundle $L \stackrel{\pi}{\longrightarrow} \Sigma$. We also embed the tube $Y \times R$ into $L$ as the complement to the zero section by the map

$$
\begin{gathered}
\Phi: Y \times R \longrightarrow L \\
(y, t) \mapsto \Phi(y, t)=\left(y, r(t)=e^{t}\right),
\end{gathered}
$$

where $r(t)$ is the radial distance in the fiber over $\pi(y)$. Using $r$ as a coordinate in an obvious way, the image of $Y \times R$ in $L$ can be written as $Y \times R^{+}$, and in the rest of this paper, we will think of $Y \times R$ and $Y \times R^{+}$as two coordinate presentations of the same underlying manifold.

Now let $g$ be a metric on $Y$, then the standard tube metric on $Y \times R$ is $(g+$ $d t \otimes d t)$. This metric is pushed forward by $\Phi$ to give a metric on its image in $L$ which is given by

$$
r^{-2}\left(r^{2} g+d r \otimes d r\right)
$$

On the other hand, we can give $L$ a complex structure. This is done simply by choosing a connection on $L$ (naturally we would like the connection to be compatible with the Hermitian metric on $L$ ). Since every connection on a Riemann surface is integrable, this is equivalent to fixing a holomorphic structure on $L$ which makes the given connection its Chern connection. The complex structure on $L$ induces a complex structure on $Y \times R^{+}$. The question then is whether or not the metric

$$
r^{-2}\left(r^{2} g+d r \otimes d r\right)
$$

or any conformally related metric is a Kaehler metric on the complex manifold $Y \times R^{+}$. With $g$ randomly chosen, one cannot expect a reasonable answer. So we restrict ourself to a class of $g$ constructed naturally from the given connection on $L$. Let $\Theta$ be a Kaehler metric on $\Sigma$, and let $\theta$ be the connection form of the given connection on $Y$. Then

$$
g=\pi^{*}(\Theta)+(-i \theta) \otimes(-i \theta)
$$

is clearly a metric on $Y$. The factor $-i$ is there because $\theta$ takes value in the Lie algebra of $U(1)$ which is $i R$.

Let $(V, w)$ be a general local complex coordinate on the Riemann surface $\Sigma$. Let $\left\{e_{h}\right\}$ and $\left\{e_{u}\right\}$ respectively be some local holomorphic gauge and unitary gauge of $L$ over $V$ and $\left(w, z_{h}\right)$ and $\left(w, z_{u}\right)$ be the corresponding local coordinates for $L$. Let $h(w)$ be the Hermitian metric form and $A_{h}$ be the connetcion form on $L$ under the gauge $e_{h}$. By some elementary calculation (see [12]), it is easy to see that

$$
\begin{aligned}
r^{2} g+d r \otimes d r= & \frac{1}{2}\left|z_{h}\right|^{2} h(w)[\partial \log h(w) \otimes \bar{\partial} \log h(w)+\bar{\partial} \log h(w) \otimes \partial \log h(w)] \\
& +\frac{1}{2} z_{h} h(w)\left[\partial \log h(w) \otimes d \bar{z}_{h}+d \bar{z}_{h} \otimes \partial \log h(w)\right] \\
& +\frac{1}{2} \bar{z}_{h} h(w)\left[d z_{h} \otimes \bar{\partial} \log h(w)+\bar{\partial} \log h(w) \otimes d z_{h}\right] \\
& +\frac{1}{2} h(w)\left[d z_{h} \otimes d \bar{z}_{h}+d \bar{z}_{h} \otimes d z_{h}\right]+r^{2} \pi^{*}(\Theta)
\end{aligned}
$$


Apparently this is a Hermitian metric, and its associated fundamental 2-form is

$$
\begin{aligned}
\Omega= & r^{2} \pi^{*}(\Theta)+\frac{i}{2}\left|z_{h}\right|^{2} h(w) A_{h} \wedge \bar{A}_{h}+\frac{i}{2} z_{h} h(w) A_{h} \wedge d \bar{z}_{h} \\
& +\frac{i}{2} \bar{z}_{h} h(w) d z_{h} \wedge \bar{A}_{h}+\frac{i}{2} h(w) d z_{h} \wedge d \bar{z}_{h} .
\end{aligned}
$$

Here, and also in the following we denote the Kaehler form of $\Theta$ by $\Theta$ itself. Thus

$$
\begin{aligned}
d \Omega= & \frac{i}{2} \bar{z}_{h} h(w) d z_{h} \wedge\left[-2 i \pi^{*}(\Theta)-\bar{F}_{h}\right] \\
& +\frac{i}{2} z_{h} h(w) d \bar{z}_{h} \wedge\left[-2 \sqrt{-1} \pi^{*}(\Theta)+F_{h}\right] .
\end{aligned}
$$

So the Kaehler condition $d \Omega=0$ reduces to

$$
\left\{\begin{array}{l}
\frac{i}{2} \bar{z}_{h} h(w) d z_{h} \wedge\left[-2 i \pi^{*}(\Theta)-\bar{F}_{h}\right]=0 \\
\frac{i}{2} z_{h} h(w) d \bar{z}_{h} \wedge\left[-2 i \pi^{*}(\Theta)+F_{h}\right]=0
\end{array}\right.
$$

i.e.

$$
F_{h}=2 i \pi^{*}(\Theta) \text {. }
$$

And if this is satisfied, then the Kaehler form of the metric is

$$
\begin{aligned}
\Omega= & \frac{i}{2}\left[-\left|z_{h}\right|^{2} h(w) F_{h}+\left|z_{h}\right|^{2} h(w) A_{h} \wedge \bar{A}_{h}+z_{h} h(w) A_{h} \wedge d \bar{z}_{h}\right. \\
& \left.+\bar{z}_{h} h(w) d z_{h} \wedge \bar{A}_{h}+h(w) d z_{h} \wedge d \bar{z}_{h}\right] \\
= & \frac{i}{2}\left[\left|z_{h}\right|^{2} \partial \bar{\partial} h(w)+z_{h} \partial h(w) \wedge d \bar{z}_{h}+\bar{z}_{h} d z_{h} \wedge \bar{\partial} h(w)+h(w) d z_{h} \wedge d \bar{z}_{h}\right] \\
= & \frac{i}{2} \partial \bar{\partial}\left[\left|z_{h}\right|^{2} h(w)\right]=\frac{i}{2} \partial \bar{\partial} r^{2} .
\end{aligned}
$$

The condition $F_{h}=2 i \pi^{*}(\Theta)$ means that $L$ is a negative line bundle. Essential examples are that $\Sigma$ is some smooth projective curve and $L$ is the pullback of the universal bundle.

More generally consider the metric $f(r)\left(r^{2} g+d r \otimes d r\right)$, where $f(r)>0$ is some function of $r$. We decide the condition of $f(r)$ for the metric to be a Kaehler metric on $\Phi(Y \times R)$. The fundamental 2-form associated with the metric is $\Omega^{\prime}=$ $f(r) \Omega$, and its differential is given by

$$
\begin{aligned}
d \Omega^{\prime}= & d f(r) \wedge \Omega+f(r) d \Omega \\
= & \bar{z}_{h} h(w) d z_{h} \wedge\left\{\left[\frac{f^{\prime}(r) r}{2}+f(r)\right] \pi^{*}(\Theta)+\frac{i}{2} f(r) F_{h}\right\} \\
& +z_{h} h(w) d \bar{z}_{h} \wedge\left\{\left[\frac{f^{\prime}(r) r}{2}+f(r)\right] \pi^{*}(\Theta)+\frac{i}{2} f(r) F_{h}\right\} .
\end{aligned}
$$


Thus the condition for the metric to be Kaehler is

$$
\left[\frac{f^{\prime}(r) r}{2}+f(r)\right] \pi^{*}(\Theta)+\frac{i}{2} f(r) F_{h}=0 .
$$

To solve this equation, choose the holomorphic structure on $L$ so that $\pi^{*}(\Theta)+$ $\frac{i}{2} F_{h}=\lambda \pi^{*}(\Theta)$, where $\lambda$ is determined by the Chern class of $L$, then the above equation reduces to

$$
\frac{f^{\prime}(r) r}{2}+\lambda f(r)=0
$$

So the solution is $f(r)=r^{-2 \lambda}$ up to a constant. Thus the condition for $f(r)\left(r^{2} g+\right.$ $d r \otimes d r)$ to be Kaehler is $f(r)=r^{-2 \lambda}$ up to a positive constant. Note that $\lambda>1$, $\lambda=1$ and $\lambda<1$ correspond respectively to the cases $L$ is positive, trivial and negative, and also that by normalizing the metric $\Theta$ on $\Sigma$, we can make $\lambda$ to be any number bigger than 1 in the case $L$ is positive, and smaller than 1 in the case $L$ is negative.

Now assume that $F_{h}$ does satisfy

$$
\pi^{*}(\Theta)+\frac{i}{2} F_{h}=\lambda \pi^{*}(\Theta)
$$

for some $\lambda>1$ or $\lambda<1$ depending on the Chern class of $L$. So $r^{-2 \lambda}\left(r^{2} g+d r \times\right.$ $d r)$ is a Kaehler metric on the complex manifold $\Phi(Y \times R)$. In terms of the complex coordinates $\left\{w, z_{h}\right\}$, its Kaehler form is

$$
\begin{aligned}
\Omega^{\prime}= & {\left[\left|z_{h}\right|^{2} h(w)\right]^{-\lambda}\left\{\frac{i\left|z_{h}\right|^{2}}{2(\lambda-1)}\left[\lambda h^{-1}(w)\left|\frac{\partial h(w)}{\partial w}\right|^{2}-\frac{\partial^{2} h(w)}{\partial w \partial \bar{w}}\right] d w \wedge d \bar{w}\right.} \\
& \left.+\frac{i}{2} z_{h} \frac{\partial h(w)}{\partial w} d w \wedge d \bar{z}_{h}+\frac{i}{2} \bar{z}_{h} \frac{\partial h(w)}{\partial \bar{w}} d z_{h} \wedge d \bar{w}+\frac{i}{2} h(w) d z_{h} \wedge d \bar{z}_{h}\right\} .
\end{aligned}
$$

We sum up the above discussion in the following lemma.

Lemma 3.1. Let $\Sigma$ be a compact Riemann surface with a Kaehler metric $\Theta$. Let $Y$ be a circle bundle over $\Sigma$. Then there is a metric $g$ on $Y$ and a holomorphic structure on $Y \times R$ such that

$$
e^{(2-2 \lambda) t}(g+d t \otimes d t)
$$

is a Kaehler metric on $Y \times R$. The complex manifold $Y \times R$ can be compactified to a complex ruled surface.

Proof. As in the above discussion, embed $Y$ as the unit circle bundle into a Hermitian line bundle $L$ and $Y \times R$ as the complement to the zero section. Choose a holomorphic structure on $L$ such that the Chern connection is a Hermitian YangMills connection, i.e.

$$
\frac{i}{2} F_{h}=(\lambda-1) \Theta
$$

The above discussion then applies to

$$
r^{-2 \lambda}\left(r^{2} g+d r \otimes d r\right)=e^{(2-2 \lambda) t}(g+d t \otimes d t) .
$$

A holomorphic line bundle $L$ has a natural complex compactification, i.e. the associated projective line bundle $S$ which is obtained by adding a point $\{\infty\}$ to 
teach fiber of $L . S$ is a ruled surface and $S \backslash(Y \times R)$ is a disjoint union of two copies of $\Sigma$, the zero section in $L$ and the section of points at infinity added to it. We shall denote the two sections by $\Sigma_{0}$ and $\Sigma_{\infty}$ respectively.

The metric we use most is the tube metric $g+d t \otimes d t=g+r^{-2} d r \otimes d r$ on $Y \times R$. It is clearly a Hermitian metric. We shall denote its associated 2-form by $\Omega_{0}$.

For later convenience, we give the explicit formula of the metric on the cotangent bundle $T^{*}(Y \times R)$ induced by $\Omega^{\prime}=r^{-2 \lambda}\left(r^{2} g+d r \otimes d r\right)$.

Lemma 3.2. The induced metric on $T^{*}(Y \times R)$ by the Kaehler metric

$$
\Omega^{\prime}=r^{-2 \lambda}\left(r^{2} g+d r \otimes d r\right)=e^{(2-2 \lambda) t}(g+d t \otimes d t)
$$

on $T_{*}(Y \times R)$ is given by

$$
\left(\Omega^{\prime}\right)^{-1}=\left|z_{h}\right|^{(2 \lambda-2)} h_{0}(w)(\Psi+\bar{\Psi}),
$$

where

$$
\begin{aligned}
\Psi= & h_{3}(w) \frac{\partial}{\partial w} \otimes \frac{\partial}{\partial \bar{w}}-z_{h} h_{2}(w) \frac{\partial}{\partial z_{h}} \otimes \frac{\partial}{\partial \bar{w}} \\
& -\bar{z}_{h} \bar{h}_{2}(w) \frac{\partial}{\partial w} \otimes \frac{\partial}{\partial \bar{z}_{h}}+\left|z_{h}\right|^{2} h_{1}(w) \frac{\partial}{\partial z_{h}} \otimes \frac{\partial}{\partial \bar{z}_{h}},
\end{aligned}
$$

and

$$
\begin{gathered}
h_{1}(w)=\frac{[h(w)]^{-\lambda}}{2(\lambda-1)}\left\{\lambda[h(w)]^{-1}\left|\frac{\partial h(w)}{\partial w}\right|^{2}-\frac{\partial^{2} h(w)}{\partial w \partial \bar{w}}\right\}, \quad h_{2}(w)=\frac{[h(w)]^{-\lambda}}{2} \frac{\partial h(w)}{\partial w} \\
h_{3}(w)=\frac{1}{2}[h(w)]^{-\lambda+1}, \quad h_{0}(w)=\left[h_{1}(w) h_{3}(w)-\left|h_{2}(w)\right|^{2}\right]^{-1}
\end{gathered}
$$

Proof. By a linear algebraic calculation.

\section{Spectral Geometry of $Y \times R$}

The successful application of the evolution equation

$$
H_{s}^{-t} \frac{\partial H_{s}^{t}}{\partial s}=-i \Lambda F_{s}
$$

to the proof of the main theorem depends crucially on certain properties of the heat kernel of the linear operator associated with the equation. For general Hermitian manifolds, the operator is $i \Lambda \bar{\partial} \partial$, where $\Lambda$ denote the adjoint to the wedge product by the fundamental form of the Hermitian metric on forms. For a Kaehler base manifold this is of course just half the ordinary Laplacian. In this section we study the heat kernel of this operator on the complex manifold $Y \times R$ with the Hermitian tube metric $g+d t \otimes d t$ as in the last section. The results obtained here may be of interest for their own sake.

We start the discussion with some simple facts on the geometry of $Y \times R$ with the Hermitian tube metric.

Recall that $\Omega_{0}$ is the fundamental 2-form associated with the tube metric. By simple calculations, we have 
Lemma 4.1. The two form $\Omega_{0}$ satisfies

$$
\begin{aligned}
& \partial \Omega_{0}=\frac{i}{2} r^{-2} \overline{z_{h}} h(w) d z_{h} \wedge F_{h}=(\lambda-1) \frac{d z_{u}}{z_{u}} \wedge \Theta, \\
& \bar{\partial} \Omega_{0}=\frac{i}{2} r^{-2} z_{h} h(w) d \bar{z}_{h} \wedge F_{h}=(\lambda-1) \frac{d \bar{z}_{u}}{\bar{z}_{u}} \wedge \Theta, \\
& \bar{\partial} \partial \Omega_{0}=0 .
\end{aligned}
$$

Let $P=i \Lambda \bar{\partial} \partial=-i \Lambda \partial \bar{\partial}$. The following two lemmas are essential to our discussion in the next section. The first one gives the relation between the operator $P$ and the Laplacian on $Y \times R$ and the second gives the formula of the heat kernel of $P$ in terms of the heat kernel of the Laplacian $\Delta_{Y}$ of $Y$ and an explicit function involving only $t$.

Lemma 4.2. Let $\Delta$ be the Laplacian of $Y \times R$ with $g+d t \otimes d t$. We have

(1) $P=\frac{1}{2} \Delta+(\lambda-1) \frac{\partial}{\partial t}$, where $t$ is the parameter of $R$ in $Y \times R$.

(2) Let $\phi=e^{2(\lambda-1) t}$, then $P \phi=0$.

Proof. (1) On any Hermitian manifold, we have

$$
\Delta f=2 P f+i *\left(\bar{\partial} \Omega^{\prime} \wedge \partial f\right)-i *\left(\partial \Omega^{\prime} \wedge \bar{\partial} f\right) .
$$

See Buchdahl [4]. It follows from Lemma 4.1 that

$$
\Delta f=2 P f+i(1-\lambda)\left(\frac{1}{\bar{z}_{u}} \frac{\partial f}{\partial z_{u}}+\frac{1}{z_{u}} \frac{\partial f}{\partial \bar{z}_{u}}\right) *\left(d z_{u} \wedge d \bar{z}_{u} \wedge \Theta\right) .
$$

Let $\{r, \theta\}$ be the polar coordinate in the fiber as in Sect 3 . It is easy to see that

$$
d z_{u} \wedge d \bar{z}_{u}=2 i r d \theta \wedge d r
$$

and

$$
\frac{1}{\bar{z}_{u}} \frac{\partial f}{\partial z_{u}}+\frac{1}{z_{u}} \frac{\partial f}{\partial \bar{z}_{u}}=\frac{1}{r} \frac{\partial f}{\partial r} .
$$

Thus

$$
\Delta f=2 P f+2(1-\lambda) \frac{\partial f}{\partial r} *(d r \wedge d \theta \wedge \Theta)=2 P f+2(1-\lambda) \frac{\partial f}{\partial t}
$$

i.e.

$$
P=\frac{1}{2} \Delta+(\lambda-1) \frac{\partial}{\partial t}
$$

(2) Simple calculation.

Lemma 4.3. Let $K_{Y}\left(x_{1}, x_{2}, s\right)$ be the heat kernel of the Laplacian $\Delta_{Y}$ of $Y$, then the heat kernel of $P$ is given by

$$
K\left[\left(x_{1}, t_{1}\right),\left(x_{2}, t_{2}\right), s\right]=K_{Y}\left(x_{1}, x_{2}, \frac{s}{2}\right) \frac{1}{(2 \pi s)^{\frac{1}{2}}} e^{-\frac{\left(t_{1}-t_{2}\right)^{2}}{2 s}+(1-\lambda)\left(t_{1}-t_{2}\right)-\frac{(\lambda-1)^{2}}{2} s} .
$$


Proof. First note that $P=\frac{1}{2} \Delta_{Y}-\frac{1}{2} \frac{\partial}{\partial t^{2}}+(\lambda-1) \frac{\partial}{\partial t}$. The formula is then derived by the usual Fourier transform method. We check that it does satisfy the requirements of a heat kernel, i.e.

(1) $\left(\frac{\partial}{\partial s}+P_{\left(x_{2}, t_{2}\right)}\right) K\left[\left(x_{1}, t_{1}\right),\left(x_{2}, t_{2}\right), s\right]=0$,

(2) For any bounded continuous function $f(x, t)$ on $Y \times R$,

$$
\lim _{s \rightarrow 0} \int_{Y \times R} K\left[\left(x_{1}, t_{1}\right),\left(x_{2}, t_{2}\right), s\right] f\left(x_{1}, t_{1}\right) d x_{1} d t_{1}=f\left(x_{2}, t_{2}\right),
$$

(3) For any bounded continuous function $f(x, t)$ on $Y \times R$,

$$
u(x, t, s)=\int_{Y \times R} K[(y, \xi),(x, t), s] f(y, \xi) d y d \xi
$$

is a solution to the heat equation

$$
\left(\frac{\partial}{\partial s}+P\right) u=0
$$

(1) can be shown by direct calculation.

To show (2), note that as $s \rightarrow 0$, the following two limits hold:

$$
\left\{\begin{array}{l}
\lim _{s \rightarrow 0} \int_{Y} K_{Y}\left(x_{1}, x_{2}, s\right) f\left(x_{1}, t_{2}\right) d x_{1}=f\left(x_{2}, t_{2}\right), \\
\lim _{s \rightarrow 0} \int_{R} \frac{1}{(2 \pi s)^{\frac{1}{2}}} e^{-\frac{\left(t_{1}-t_{2}\right)^{2}}{2 s}+(1-\lambda)\left(t_{1}-t_{2}\right)-\frac{(\lambda-1)^{2}}{2} s} f\left(x_{1}, t_{1}\right) d t_{1}=f\left(x_{1}, t_{2}\right) .
\end{array}\right.
$$

The second limit is uniform for $x_{1}$ as $Y$ is compact. (2) then follows easily.

Finally, we note that the differentiations can be carried out under integration, since the integration of the results of the differentiations involved converge absolutely and uniformly. (3) then follows from (1).

The key property of the heat kernel of $P$ needed to construct a solution to the Hermitian Yang-Mills equation by the evolution equation method is the following lemma.

Lemma 4.4. Let $f$ be a function on $Y \times R$ such that

$$
|f|=O\left(e^{-|t|}\right), \quad t \rightarrow \pm \infty .
$$

Let

$$
G(t)=\int_{0}^{\infty} \frac{1}{\sqrt{2 \pi s}} e^{-\frac{t^{2}}{2 s}+(1-\lambda) t-\frac{(1-\lambda)^{2}}{2} s} d s
$$

and

$$
u\left(t_{2}\right)=\int_{0}^{\infty} d s \int_{Y \times R} K\left[\left(x_{1}, t_{1}\right),\left(x_{2}, t_{2}\right), s\right]\left|f\left(x_{1}, t_{1}\right)\right| d x_{1} d t_{1} .
$$

The following two conclusions hold for $\delta_{\lambda}=\min \{1,2|1-\lambda|\}$ :

(1) If $\lambda<1$, then

$$
G(t)= \begin{cases}\frac{1}{(1-\lambda)}, & t \geqq 0 \\ \frac{1}{(1-\lambda)} e^{2(1-\lambda) t}, & t<0,\end{cases}
$$


and

$$
u\left(t_{2}\right)= \begin{cases}O\left(e^{-\delta_{\lambda} t_{2}}\right), & t_{2} \longrightarrow+\infty \\ O(1), & t_{2} \longrightarrow-\infty\end{cases}
$$

(2) If $\lambda>1$, then

$$
G(t)= \begin{cases}\frac{1}{(\lambda-1)} e^{2(1-\lambda) t}, & t \geqq 0 \\ \frac{1}{(\lambda-1)}, & t \leqq 0,\end{cases}
$$

and

$$
u\left(t_{2}\right)= \begin{cases}O(1), & t_{2} \longrightarrow+\infty \\ O\left(e^{\delta_{\lambda} t_{2}}\right), & t_{2} \longrightarrow-\infty .\end{cases}
$$

Proof. We show (1). (2) follows similarly.

First we note that

$$
G(t)=e^{(1-\lambda) t} g(t),
$$

where $g(t)=\int_{0}^{\infty} \frac{1}{\sqrt{2 \pi s}} e^{-\frac{t^{2}}{2 s}-\frac{(1-\lambda)^{2}}{2} s} d s=g(-t)$. Thus to prove the first result, it suffices to prove the case $t \geqq 0$, in which we write

$$
G(t)=\left(\int_{0}^{\frac{t}{(1-\lambda)}}+\int_{\frac{t}{(1-\lambda)}}^{\infty}\right) \frac{1}{\sqrt{2 \pi s}} e^{-\frac{[t-(1-\lambda) s]^{2}}{2 s}} d s .
$$

Let $s^{\prime}=\frac{[t-(1-\lambda) s]^{2}}{2 s}$. By simple calculation, we have

$G(t)=\frac{1}{\sqrt{2 \pi}(1-\lambda)}$

$$
\times \int_{0}^{\infty} \frac{\sqrt{s^{\prime}+(1-\lambda) t-\sqrt{s^{\prime 2}+2(1-\lambda) t s^{\prime}}}+\sqrt{s^{\prime}+(1-\lambda) t+\sqrt{s^{\prime 2}+2(1-\lambda) t s^{\prime}}}}{\sqrt{s^{\prime 2}+2(1-\lambda) t s^{\prime}}} e^{-s^{\prime}} d s^{\prime}
$$

$=\frac{1}{\sqrt{2 \pi}(1-\lambda)} \int_{0}^{\infty} \frac{\sqrt{2}}{\sqrt{s^{\prime}}} e^{-s^{\prime}} d s^{\prime}=\frac{1}{(1-\lambda)}$.

We have used in the above proof the following simple fact:

$$
\begin{aligned}
\sqrt{s^{\prime}} & +(1-\lambda) t-\sqrt{s^{\prime 2}+2(1-\lambda) t s^{\prime}} \\
& =\sqrt{2} \sqrt{s^{\prime}+(1-\lambda) t+\sqrt{s^{\prime 2}+2(1-\lambda) t s^{\prime}}}
\end{aligned}
$$

To prove the second result, we note that by the hypothesis,

$$
\int_{Y} K_{Y}\left(x_{1}, x_{2}, s\right)\left|f\left(x_{1}, t_{1}\right)\right| d x_{1} \leqq C \cdot e^{-\left|t_{1}\right|} .
$$


It follows that

$$
\begin{aligned}
u\left(t_{2}\right) & =\int_{0}^{\infty} d s \int_{Y \times R} K\left[\left(x_{1}, t_{1}\right),\left(x_{2}, t_{2}\right), s\right]\left|f\left(x_{1}, t_{1}\right)\right| d x_{1} d t_{1} \\
& \leqq C \cdot \int_{0}^{\infty} d s \int_{R} \frac{1}{\sqrt{2 \pi s}} e^{-\frac{\left(t_{1}-t_{2}\right)^{2}}{2 s}+(1-\lambda)\left(t_{1}-t_{2}\right)-\frac{(\lambda-1)^{2}}{2} s} e^{-\left|t_{1}\right|} d t_{1} \\
& =C \cdot \int_{R} e^{-\left|t_{1}\right|} d t_{1} \int_{0}^{\infty} \frac{1}{\sqrt{2 \pi s}} e^{-\frac{\left(t_{1}-t_{2}\right)^{2}}{2 s}+(1-\lambda)\left(t_{1}-t_{2}\right)-\frac{(1-\lambda)^{2} s}{2}} d s \\
& =C \cdot \int_{R} G\left(t_{1}-t_{2}\right) e^{-\left|t_{1}\right|} d t_{1} \\
& =C \cdot \int_{-\infty}^{t_{2}} \frac{1}{(1-\lambda)} e^{2(1-\lambda)\left(t_{1}-t_{2}\right)} e^{-\left|t_{1}\right|} d t_{1}+C \cdot \int_{t_{2}}^{\infty} \frac{1}{(1-\lambda)} e^{-\left|t_{1}\right|} d t_{1} .
\end{aligned}
$$

Thus if $t_{2} \geqq 0$, then

$$
\begin{aligned}
u\left(t_{2}\right) & \leqq \frac{C}{(1-\lambda)} e^{-2(1-\lambda) t_{2}}\left[\int_{-\infty}^{0} e^{(3-2 \lambda) t_{1}} d t_{1}+\int_{0}^{t_{2}} e^{(1-2 \lambda) t_{1}} d t_{1}\right]+\frac{C}{(1-\lambda)} \int_{t_{2}}^{\infty} e^{-t_{1}} d t_{1} \\
& =\frac{2 C}{(1-2 \lambda)}\left[e^{-t_{2}}-\frac{1}{(1-\lambda)(3-2 \lambda)} e^{-2(1-\lambda) t_{2}}\right]
\end{aligned}
$$

and $t_{2}<0$, then

$$
\begin{aligned}
u\left(t_{2}\right) & \leqq \frac{C}{(1-\lambda)}\left[e^{-2(1-\lambda) t_{2}} \int_{-\infty}^{t_{2}} e^{2(1-\lambda) t_{1}+t_{1}} d t_{1}+\int_{t_{2}}^{0} e^{t_{1}} d t_{1}+\int_{0}^{\infty} e^{-t_{1}} d t_{1}\right] \\
& =\frac{C}{(1-\lambda)}\left[2-\frac{(2-2 \lambda)}{(3-2 \lambda)} e^{t_{2}}\right] .
\end{aligned}
$$

The result then follows easily.

The next two lemmas assert that the maximum principle holds for the operator $P$, both in the elliptic case and in the parabolic case. These will be needed in the next section to establish the existence and the uniqueness of the solution to the evolution equation.

Lemma 4.5. (The maximum principle, elliptic case). Let $D$ be a compact domain in $Y \times R$ and let $u$ be a function on $D$ such that

$$
P u \leqq 0 \text {. }
$$

Then $u$ achieves its maximum on the boundary of $D$.

Lemma 4.6. (The maximum principle, parabolic case). Let $v(\cdot, s)$ be a function defined on $D \times[a, b)$ such that

$$
\left(\frac{\partial}{\partial s}+P\right) v \leqq 0 .
$$

Then $v$ achieves its maximum on the boundary of $D \times[a, b)$. 
Proof of Lemmas 4.5 and 4.6. We note that $P$ differs from $\frac{1}{2} \Delta$ by a first order term $(k-1) \frac{\partial}{\partial t}$, which vanishes at an inner point of maximum value, should such a point exist. Thus the proof of the maximum principle for ordinary Laplacian works for our case. See $[11,16]$.

Remark. It follows plainly from the proof of the maximum principle that if $u$ (resp. $v$ ) are non-negative functions, then the conclusion of Lemma 4.5 (resp. Lemma 4.6) still holds if

$$
P u \leqq 0 \quad\left(\operatorname{resp} .\left(\frac{\partial}{\partial s}+P\right) v \leqq 0\right)
$$

whenever $u>0$ (resp. $v>0$ ). Thus we can, as we do in the following, apply the lemmas to the function $|i \Lambda F(s)|$ which may not be smooth at points where it vanishes.

\section{The Existence Theorem}

In this section we prove Theorem 2.1 and then describe how Theorem 2.1 gives a map from $\mathcal{M}_{c}^{*}(+,+)$ to the set of gauge equivalence classes of ASD connections on $Y \times R$.

Given a holomorphic bundle on $S$ representing an element of $\mathcal{M}_{c}(+,+)$, we deform a suitable initial Hermitian metric on the bundle to a desired one, through special paths defined by the solution to the evolution equation

$$
H_{s}^{-t} \frac{\partial H_{s}^{t}}{\partial s}=-i \Lambda F_{s}
$$

using the tube metric on $Y \times R$.

We restrict ourselves in Theorem 2.1 to holomorphic bundles of rank two and trivial first Chern class. However it will be clear from the proof of the theorem that the results of the theorem hold without these restrictions on rank and first Chern class.

For a holomorphic Hermitian bundle on a Hermitian manifold, let $P_{A}=i \Lambda \bar{\partial} \partial_{A}$ and $\bar{P}_{A}=-i \Lambda \partial_{A} \bar{\partial}$, in addition to $P=i \Lambda \bar{\partial} \partial=-i \Lambda \partial \bar{\partial}$.

We now begin the proof of Theorem 2.1. We suppose $c_{1}(Y)<0$. The proof for the other case is almost identical. First we prove the uniqueness part of Theorem 2.1.

Proposition 5.1. Let $\mathcal{E}$ be a bundle as in Theorem 2.1 and $H_{1}$ and $H_{2}$ be two Hermitian metrics on $\mathcal{E}$. If $H_{1}$ and $H_{2}$ both satisfy (1) and (2) of Theorem 2.1, then $H_{1}=H_{2}$.

Proof. Let $h$ be the endomorphism of $\mathcal{E}$ determined by

$$
\langle\xi, \eta\rangle_{H_{2}}=\langle h \xi, \eta\rangle_{H_{1}} .
$$

Then $h$ is positive self-adjoint with respect to both $H_{1}$ and $H_{2}$. Let $\sigma\left(H_{1}, H_{2}\right)=$ $\operatorname{tr}(h)+\operatorname{tr}\left(h^{-1}\right)-2 r$, where $r$ is the rank of $\mathcal{E}$. If

$$
h=\left(\begin{array}{ccc}
\lambda_{1} & & \\
& \ddots & \\
& & \lambda_{r}
\end{array}\right)
$$


then

$$
\sigma\left(H_{1}, H_{2}\right)=\sum_{i}\left(\lambda_{i}+\lambda_{i}^{-1}\right)-2 r \geqq 0,
$$

and the equality holds iff $\lambda_{i}=1, i=1, \ldots, r$. Thus it suffices to show that $\sigma\left(H_{1}, H_{2}\right)=0$.

Locally under a frame $H_{2}=h^{t} H_{1}$, so we have

$$
\begin{aligned}
A_{2} & =H_{2}^{-t} \partial H_{2}^{t}=h^{-1} A_{1} h+h^{-1} \partial h \\
& =A_{1}+h^{-1}\left[\partial h+A_{1} h-h A_{1}\right]=A_{1}+h^{-1} \partial_{A_{1}} h,
\end{aligned}
$$

thus

$$
F_{2}=F_{1}+\bar{\partial}\left(h^{-1} \partial_{A_{1}} h\right)
$$

By the hypothesis,

$$
i \Lambda F_{1}=i \Lambda F_{2}=0 \text {. }
$$

It follows that

$$
i \Lambda h^{-1} \bar{\partial} \partial_{A_{1}} h-i \Lambda h^{-1}(\bar{\partial} h) h^{-1} \wedge \partial_{A_{1}} h=0 .
$$

Multiplying it by $h$ on the left, then taking the trace, we get

$$
i \Lambda \bar{\partial} \partial(\operatorname{tr} h)=i \Lambda \operatorname{tr}\left[(\bar{\partial} h) h^{-1} \wedge \partial_{A_{1}} h\right] .
$$

The right-hand side of the above equation is negative. To see this, for any point $x \in Y \times R$, let $\left(e_{1}, \ldots, e_{r}\right)$ be a local frame of the bundle around $x$, unitary with respect to $H_{1}$, such that the matrix representation of $h$ under this frame is diagonal at $x$, say $\left(\begin{array}{ccc}\lambda_{1} & & \\ & \ddots & \\ & & \lambda_{r}\end{array}\right), \lambda_{i}>0$, and the connection form of $A_{1}$ vanishes at $x$, then at this point

$$
\begin{aligned}
i \Lambda \operatorname{tr}\left[(\bar{\partial} h) h^{-1} \wedge \partial_{A_{1}} h\right] & =i \Lambda \operatorname{tr}\left[(\bar{\partial} h)\left(\begin{array}{ccc}
\lambda_{1}^{-1} & \\
& \cdots & \\
& & \lambda_{r}^{-1}
\end{array}\right) \wedge \partial h\right] \\
& =\sum_{i, j} i \Lambda \lambda_{j}^{-1} \bar{\partial} h_{i j} \wedge \partial h_{j i} \\
& =-\sum_{j} \lambda_{j}^{-1} \sum_{i}\left|\partial h_{j i}\right|^{2} \leqq 0 .
\end{aligned}
$$

We have used the facts that $h_{i j}=\overline{h_{j i}}$, and that for a $(1,0)$-form $\phi, i \Lambda \bar{\phi} \wedge \phi=-|\phi|^{2}$. It follows that

$$
P \operatorname{tr}(h) \leqq 0
$$

Similarly,

$$
P \operatorname{tr}\left(h^{-1}\right) \leqq 0
$$

Therefore

$$
P \sigma\left(H_{1}, H_{2}\right) \leqq 0
$$


On the other hand, note that $\sigma$ and the metric $d$ are equivalent in a neighbourhood of zero, i.e. there are constants $c_{1}, c_{2}>0$ such that for $d$ sufficiently small,

$$
c_{1} d^{2} \leqq \sigma \leqq c_{2} d^{2}
$$

By the hypothesis, $H_{1}$ and $H_{2}$ both satisfy (2) of Theorem 2.1. It follows that

$$
\sigma\left(H_{1}, H_{2}\right) \leqq C
$$

and

$$
\sigma\left(H_{1}, H_{2}\right)(y, t) \longrightarrow 0, \quad t \rightarrow \infty
$$

Let

$$
\phi=e^{2(\lambda-1) t}
$$

and let

$$
\psi=\sigma\left(H_{1}, H_{2}\right)-\frac{C \cdot \phi}{e^{-2(\lambda-1) i}} .
$$

The above and (2) of Lemma 4.2 imply that

$$
P \psi \leqq 0 \text {. }
$$

Consider the function $\psi$ on the domain $D_{i j}=Y \times[-i, j]$ in $Y \times R$. On the boundary $\partial D_{i j}=Y \times\{-i\} \cup Y \times\{j\}$,

$$
\psi \leqq \max \left\{0, \sigma\left(H_{1}, H_{2}\right)(y, j)-C e^{2(\lambda-1)(i+j)}\right\} .
$$

It follows from the maximum principle in Lemma 4.6 that

$$
\sigma\left(H_{1}, H_{2}\right) \leqq \frac{C \cdot \phi}{e^{-2(\lambda-1) i}}+\max \left\{0, \sigma\left(H_{1}, H_{2}\right)(y, j)-C e^{2(\lambda-1)(i+j)}\right\},
$$

independent of $i, j$. As $i$ and $j$ tend to $\infty$, the right-hand side tends to 0 , thus

$$
\sigma\left(H_{1}, H_{2}\right)=0 \text {. }
$$

This completes the proof of Proposition 5.1, i.e. the uniqueness part of Theorem 2.1 .

We now move on to prove the existence part of Theorem 2.1. We need a suitable Hermitian metric on $\mathcal{E}$ to start with.

Lemma 5.2. Let $\mathcal{E}$ be a holomorphic bundle as in Theorem 2.1. Let $H_{0}^{\prime}$ be a smooth Hermitian metric on $\mathcal{E}$ which extends the given unitary structures of $\mathcal{E}$ over $\Sigma_{0}$ and $\Sigma_{\infty}$. Then with respect to the Hermitian tube metric $\Omega_{0}=(g+d t \otimes d t)$, the curvature $F_{H_{0}^{\prime}}$ of the Chern connection of $H_{0}^{\prime}$ satisfies

$$
\left|F_{H_{0}^{\prime}}\right|_{\Omega_{0}}=O\left(e^{-|t|}\right), \quad t \rightarrow \pm \infty
$$

and

$$
\begin{gathered}
\int_{0}^{\infty} d s \int_{Y \times R} K\left[\left(x_{1}, t_{1}\right),\left(x_{2}, t_{2}\right), s\right]\left|i \Lambda F_{H_{0}^{\prime}}\left(x_{1}, t_{1}\right)\right| d x_{1} d t_{1} \\
\quad= \begin{cases}O\left(e^{-\delta_{\lambda} t_{2}}\right), & t_{2} \longrightarrow+\infty \\
O(1), & t_{2} \longrightarrow-\infty\end{cases}
\end{gathered}
$$

Here $\delta_{\lambda}$ is the positive number defined in Lemma 4.4 . 
Proof. As in Sect. 3, let $(z, w)$ be holomorphic coordinates in some neighbourhood $U$ of some point at the end $\Sigma_{0}$, say. Thus $U \cap \Sigma_{0}=\{z=0\}$. Choose a local holomorphic trivialization of $\mathcal{E}$ over the neighbourhood. Then the curvature $F_{H_{0}^{\prime}}$ is a matrix valued $(1,1)$-form and can be written as

$$
F_{H_{0}^{\prime}}=F_{11} d z \wedge d \bar{z}+F_{12} d z \wedge d \bar{w}+F_{21} d w \wedge d \bar{z}+F_{22} d w \wedge d \bar{w} .
$$

Since the bundle $\mathcal{E}$ is flat over the end,

$$
F_{22}(0, w)=0 \text {. }
$$

By Taylor expansion,

$$
\left|F_{22}(z, w)\right|=O(|z|), \quad|z| \longrightarrow 0 .
$$

On the other hand, we have, by the formula in Lemma 3.2,

$$
\begin{array}{ll}
|d z|_{\Omega_{0}},|d \bar{z}|_{\Omega_{0}}=O(|z|), & |z| \rightarrow 0, \\
|d w|_{\Omega_{0}},|d \bar{w}|_{\Omega_{0}}=O(1), & |z| \rightarrow 0 .
\end{array}
$$

It follows that in any compact subset of the neighbourhood,

$$
\left|F_{H_{0}^{\prime}}\right|_{\Omega_{0}}=O(|z|)=O\left(e^{-|t|}\right), \quad|t| \rightarrow \infty .
$$

But this holds for the whole neighbourhood of the end, since we can always choose a finite number of such neighbourhoods to cover the end.

The second assertion follows from the first assertion and (1) of Lemma 4.4, recalling that we assume $c_{1}(Y)<0$ here.

Remark. By the proof of this lemma, it follows that if $e$ is a local holomorphic frame in a neighbourhood of some point at the end $\Sigma_{0}$ or $\Sigma_{\infty}$ such that the restriction of $e$ over the end is flat in the obvious sense, then the connection 1-form under this frame $A_{H_{0}^{\prime}}$, its covariant differential $\nabla\left(A_{H_{0}^{\prime}}\right)$ and the covariant differential of the curvature form $\nabla\left(F_{H_{0}^{\prime}}\right)$, viewed as matrix-valued forms, satisfy

$$
\begin{aligned}
\left|A_{H_{0}^{\prime}}\right|_{\Omega_{0}}=O\left(e^{-|t|}\right), \quad t \rightarrow \pm \infty, \\
\left|\nabla\left(A_{H_{0}^{\prime}}\right)\right|_{\Omega_{0}}=O\left(e^{-|t|}\right), \quad t \rightarrow \pm \infty, \\
\left|\nabla\left(F_{H_{0}^{\prime}}\right)\right|_{\Omega_{0}}=O\left(e^{-|t|}\right), \quad t \rightarrow \pm \infty .
\end{aligned}
$$

Actually all the higher derivatives decay in a similar way over the ends.

Starting with $H_{0}^{\prime}$, we shall now construct a Hermitian metric $H$ on $\left.\mathcal{E}\right|_{Y \times R}$ which satisfies

$$
\left\{\begin{array}{l}
i \Lambda F_{H}=0 \\
d\left(H, H_{0}^{\prime}\right) \leqq C \\
d\left(H, H_{0}^{\prime}\right)(y, t)=O\left(e^{-\delta_{\lambda} t}\right), \quad t \longrightarrow \infty
\end{array}\right.
$$

using the evolution equation

$$
H^{-t}(s) \frac{\partial H^{t}(s)}{\partial s}=-i \Lambda F(s)
$$

of Hermitian metrics. 
Consider the Dirichlet boundary problem

$$
(* *)\left\{\begin{array}{l}
H_{i j}^{-t}(s) \frac{\partial H_{i j}^{t}(s)}{\partial s}=-i \Lambda F(s) \\
H_{i j}(0)=H_{0}^{\prime} \\
\left.H_{i j}(s)\right|_{\partial D_{i j}}=\left.H_{0}^{\prime}\right|_{\partial D_{i j}}
\end{array}\right.
$$

for Hermitian metrics on $\left.\mathcal{E}\right|_{D_{i j}}$, where $D_{i j}$ is the compact domain $Y \times(-i, j)$ in $Y \times R$ for $i, j \in N$. This is a nonliear parabolic equation. Its linearization is

$$
\left(\frac{\partial}{\partial s}+P_{A(s)}\right) u=0 .
$$

We refer to Sect. 6 of [19] for the fact that on each $D_{i j}$ there is a unique solution $H_{i j}(s), 0 \leqq s<\infty$ to $(* *)$.

We now show that for each pair $i, j, H_{i j}(s)$ converges to some $H_{i j}$ on $\left.\mathcal{E}\right|_{\bar{D}_{i j}}$ as $s \rightarrow \infty$. We need the following lemma.

Lemma 5.3. For a solution $H(s)$ to the evolution equation

$$
H^{-t}(s) \frac{\partial H^{t}(s)}{\partial s}=-i \Lambda F(s)
$$

on any Hermitian manifold, we have $\left(\frac{\partial}{\partial s}+P\right)\left|i \Lambda F_{s}\right| \leqq 0$ whenever $i \Lambda F(s) \neq 0$.

Proof. The norm $|\cdot|$ depends on the parameter $s$. Nevertheless, since $i \Lambda F(s)$ is always self-adjoint with respect to $H(s)$, we see that

$$
|i \Lambda F(s)|^{2}=\operatorname{Tr}(i \Lambda F(s) \wedge i \Lambda F(s)) .
$$

The right-hand side is an expression independent of the norm. This simplifies the calculation of the $\frac{\partial}{\partial s}$ component of derivatives. A simple calculation then shows that

$$
\frac{\partial}{\partial s}|i \Lambda F(s)|^{2}=-\left\langle P_{A(s)}(i \Lambda F(s)), i \Lambda F(s)\right\rangle-\left\langle i \Lambda F(s), \bar{P}_{A(s)}(i \Lambda F(s))\right\rangle .
$$

On the other hand, we have in general

$$
\left\{\begin{array}{l}
P|\alpha|^{2}=\left\langle P_{A} \alpha, \alpha\right\rangle+\left\langle\alpha, \bar{P}_{A} \alpha\right\rangle-\left|\nabla_{A} \alpha\right|^{2} \\
P|\alpha|^{2}=2|\alpha| P|\alpha|-|d| \alpha||^{2},
\end{array}\right.
$$

whenever $\alpha \neq 0$. Let $\alpha=i \Lambda F(s)$ and $\nabla=\nabla_{A(s)}$, then we see that

$$
2|i \Lambda F(s)|\left(\frac{\partial}{\partial s}+P\right)|i \Lambda F(s)|=|d| i \Lambda F(s)||^{2}-\left|\nabla_{A(s)}(i \Lambda F(s))\right|^{2},
$$

whenever $i \Lambda F(s) \neq 0$. The result then follows from Kato's inequality,

$$
|d| i \Lambda F(s)||^{2} \leqq\left|\nabla_{A(s)}(i \Lambda F(s))\right|^{2} .
$$


By this lemma and the remark at the end of the last section, we have,

$$
\left|H_{i j}^{-t}(s) \frac{\partial H_{i j}^{t}(s)}{\partial s}\right|=\left|i \Lambda F_{i j}(s)\right| \leqq \int_{D_{i j}} K_{D_{l j}}(s, x, y)\left|i \Lambda F_{H_{0}^{\prime}}(y)\right| d y,
$$

where $K_{D_{l j}}(s, x, y)$ is the heat kernel ([5]) of $P$ on $D_{i j}$ with Dirichlet boundary condition. Now the maximum principle implies [5] that

$$
K_{D_{i j}}(s, x, y) \leqq K(s, x, y),
$$

where $K(s, x, y)$ is the heat kernel of $P$ on $Y \times R$ as in the last section. Thus furthermore we have

$$
\begin{aligned}
\int_{0}^{\infty}\left|H_{i j}^{-t}(s) \frac{\partial H_{i j}^{t}(s)}{\partial s}\right| d s & \leqq \int_{0}^{\infty} d s \int_{D_{i j}} K_{D_{l j}}(s, x, y)\left|i \Lambda F_{0}(y)\right| d y \\
& \leqq \int_{0}^{\infty} d s \int_{Y \times R} K(s, x, y)\left|i \Lambda F_{0}(y)\right| d y
\end{aligned}
$$

By Lemma 4.4,

$$
\int_{0}^{\infty}\left|H_{i j}^{-t}(s) \frac{\partial H_{i j}^{t}(s)}{\partial s}\right| d s=O(1),
$$

so for any given $\varepsilon>0$, if $s_{1}, s_{2}>0$ are sufficiently large, then

$$
\int_{s_{1}}^{s_{2}}\left|H_{i j}^{-t}(s) \frac{\partial H_{i j}^{t}(s)}{\partial s}\right| d s \leqq \varepsilon
$$

Hence we have

$$
d\left(H_{i j}\left(s_{1}\right), H_{i j}\left(s_{2}\right)\right) \leqq \int_{s_{1}}^{s_{2}}\left|H_{i j}^{-t}(s) \frac{\partial H_{i j}^{t}(s)}{\partial s}\right| d s \leqq \varepsilon,
$$

where $d\left(H_{i j}\left(s_{1}\right), H_{i j}\left(s_{2}\right)\right)$ denotes the distance between $H_{i j}\left(s_{1}\right)$ and $H_{i j}\left(s_{2}\right)$ in the space of Hermitian metrics with the complete metric as above. Thus $H_{i j}(s)$ converges in $C^{0}$ to some $H_{i j}$ on $\left.\Pi^{*}(E)\right|_{\bar{D}_{i j}}$. (As the referee pointed out, the preceding arguments are basically the same as those used by Donaldson in [7], Sect. 2.4.)

Now by Lemma 6.4 in [19] and the remark following it, we know that $H_{i j}(s)$ are bounded in $L_{2, l o c}^{p}$, i.e. bounded in $L_{2}^{p}$ over any compact subset of $D_{i j}(s)$. Therefore there is a sequence $\left\{s_{l}\right\}, l \rightarrow \infty$ such that $H_{i j}\left(s_{l}\right)$ converges, as $l \rightarrow \infty$, in $C^{1}$ and also weakly in $L_{2}^{p}$ to some $H_{i j}(\infty)$ which is necessarily $H_{i j}$ on $\left.\Pi^{*}(E)\right|_{D_{l j}}$. Since $\left|i \Lambda F_{H_{i j}\left(s_{l}\right)}\right| \rightarrow 0$ as $l \rightarrow \infty, i \Lambda F_{H_{i j}}=0$ weakly. By elliptic regularity, $H_{i j}$ is smooth and satisfies $i \Lambda F_{H_{\imath}}=0$ in the normal sense. Clearly.

$$
\left.H_{i j}\right|_{\partial D_{\imath \jmath}}=\left.H_{0}^{\prime}\right|_{\partial D_{\imath \jmath}} .
$$

Next we show that, for each fixed $i$, the sequence $\left\{H_{i j}, j=1, \ldots,\right\}$ converges in $C^{0}$ on any compact domain of $D_{i}=Y \times(-i, \infty)$ to a Hermitian metric $H_{i}$ on $\left.\mathcal{E}\right|_{D_{l}}$ which satisfies

$$
\left\{\begin{array}{l}
i \Lambda F_{i}=0 \\
\left.H_{i}\right|_{\partial D_{\imath}}=\left.H_{0}^{\prime}\right|_{\partial D_{\imath}} .
\end{array}\right.
$$


Consider again the pointwise distance $d\left(H_{i j}, H_{i k}\right)$ between $H_{i j}$ and $H_{i k}$ for any positive integers $j, k, j<k$ say. We have

$$
\begin{aligned}
d\left(H_{i j}, H_{j k}\right) & \leqq d\left(H_{i j}, H_{0}^{\prime}\right)+d\left(H_{0}^{\prime}, H_{j k}\right) \\
& \leqq \lim _{s \rightarrow \infty}\left[d\left(H_{i j}(s), H_{0}^{\prime}\right)+d\left(H_{0}^{\prime}, H_{i k}(s)\right)\right] \\
& \leqq \int_{o}^{\infty}\left[\left|H_{i j}^{-t}(s) \frac{\partial H_{i j}^{t}(s)}{\partial s}\right|+\left|H_{i k}^{-t}(s) \frac{\partial H_{i k}(s)}{\partial s}\right|\right] d s \\
& \leqq 2 \int_{0}^{\infty} d s \int_{Y \times R} K(s, x, y)\left|i \Lambda F_{H_{0}^{\prime}}(y)\right| d y .
\end{aligned}
$$

Again by Lemma 4.4,

$$
\max _{\partial D_{i j}} d\left(H_{i j}, H_{i k}\right)(x) \leqq C \cdot e^{-\delta_{\lambda j}} .
$$

On the other hand, for the function

$$
\sigma\left(H_{i j}, H_{i k}\right)=\operatorname{tr}\left(H_{i j}^{-1} H_{i k}\right)+\operatorname{tr}\left(H_{i k}^{-1} H_{i j}\right)-2 r
$$

defined on $D_{i j}$, we have shown in the proof of Proposition 5.1 that

$$
P \sigma\left(H_{i j}, H_{i k}\right) \leqq 0 \text {. }
$$

By the maximum principle,

$$
\sigma\left(H_{i j}, H_{i k}\right)(x) \leqq \max _{\partial D_{i j}} \sigma\left(H_{i j}, H_{i k}\right), \quad x \in D_{i j} .
$$

Now $\sigma$ and $d$ are equivalent in a neighbourhood of zero, i.e. there are constants $c_{1}, c_{2}>0$ such that for $d$ sufficiently small,

$$
c_{1} d^{2} \leqq \sigma \leqq c_{2} d^{2}
$$

Thus for $j$ sufficiently large, we have

$$
\sigma\left(H_{i j}, H_{i k}\right)(x) \leqq \max _{\partial D_{i j}} \sigma\left(H_{i j}, H_{i k}\right) \leqq C^{\prime} e^{-2 \delta_{\lambda j}},
$$

for some constant $C^{\prime}>0$. Again since $\sigma$ is equivalent to $d$, we see that $\left\{H_{i j}\right\}$ converges in $C^{0}$ to some Hermitian metric, $H_{i}$ say, on $\left.\Pi^{*}(E)\right|_{D_{i}}$.

By the discussions above, we see again that $\left\{H_{i j}\right\}$, or a subsequence of it, converges weakly in $L_{2}^{p}$ to $H_{i}$ on any compact subset of $D_{i}$ and $H_{i}$ is smooth with

$$
i \Lambda F_{H_{l}}=\lim _{i \rightarrow \infty} i \Lambda F_{i j}=0
$$

Moreover, since

$$
\begin{aligned}
d\left(H_{0}^{\prime}, H_{i}\right) & =\lim _{j \rightarrow \infty} d\left(H_{0}^{\prime}, H_{i j}\right) \leqq \int_{0}^{\infty}\left|H_{i j}^{-t}(s) \frac{\partial H_{i j}^{t}(s)}{\partial s}\right| d s \\
& \leqq \int_{0}^{\infty} d s \int_{Y \times R} K\left[\left(y_{1}, t_{1}\right),\left(y_{2}, t_{2}\right), s\right]\left|i \Lambda F_{H_{0}^{\prime}}\left(y_{1}, t_{1}\right)\right| d y_{1} d t_{1}
\end{aligned}
$$


It follows from Lemma 5.2 and Lemma 4.4 that

$$
d\left(H_{0}^{\prime}, H_{i}\right)=O\left(e^{-\delta_{\lambda} t}\right), \quad t \longrightarrow \infty,
$$

and the bound $O\left(e^{-\delta_{i} t}\right)$ can be chosen to be independent of $i$.

Finally, we now show that as $i \rightarrow \infty$, the sequence $\left\{H_{i}\right\}$ converges in $C^{0}$ to a Hermitian metric $H$ on $\left.\mathcal{E}\right|_{Y \times R}$ and $H$ satisfies

$$
\left\{\begin{array}{l}
i \Lambda F_{H}=0 \\
d\left(H, H_{0}^{\prime}\right) \leqq C \\
d\left(H, H_{0}^{\prime}\right)(y, t)=O\left(e^{-\delta_{k} t}\right), \quad t \longrightarrow \infty
\end{array}\right.
$$

As before, for any integers $i, k, i<k$ say, we have

$$
\Delta \sigma\left(H_{i}, H_{k}\right) \leqq 0 \text {. }
$$

On the other hand,

$$
\begin{aligned}
d\left(H_{i}, H_{k}\right) & \leqq d\left(H_{i}, H_{0}^{\prime}\right)+d\left(H_{k}, H_{0}^{\prime}\right) \\
& \leqq \lim _{j \rightarrow \infty}\left[d\left(H_{i j}, H_{0}^{\prime}\right)+d\left(H_{k j}, H_{0}^{\prime}\right)\right] \\
& \leqq \int_{0}^{\infty}\left[\left|H_{i j}^{-t}(s) \frac{\partial H_{i j}^{t}(s)}{\partial s}\right|+\left|H_{k j}^{-t}(s) \frac{\partial H_{k j}^{t}(s)}{\partial s}\right|\right] d s \\
& \leqq \int_{0}^{\infty} d s \int_{Y \times R} K\left[\left(y_{1}, t_{1}\right),\left(y_{2}, t_{2}\right), s\right]\left|i \Lambda F_{H_{0}^{\prime}}\left(y_{1}, t_{1}\right)\right| d y_{1} d t_{1} \leqq C
\end{aligned}
$$

by Lemma 5.2 . Here as before $C$ is a general constant independent of $i, k$.

Now consider the function

$$
\psi=\sigma\left(H_{i}, H_{k}\right)-\frac{C^{\prime}}{e^{-2(\lambda-1) i}} \phi
$$

on $D_{i}$, where $\phi=e^{2(\lambda-1) t}$. By (2) of Lemma 4.2, $P \psi \leqq 0$. Choose $C^{\prime}$ large, $C^{\prime}=e^{C}$ say, then $\psi \leqq 0$ on $\partial D_{i}$ and that $\lim _{t \rightarrow \infty} \psi=0$. Thus by the maximum principle, we have

$$
\psi \leqq 0
$$

i.e.

$$
\sigma\left(H_{i}, H_{k}\right) \leqq \frac{C^{\prime}}{e^{-2(\lambda-1) i}} \phi .
$$

It follows that $H_{i}$ converges in $C^{0}$ to some Hermitian metric $H$ on $\left.\mathcal{E}\right|_{Y \times R}$ as $i$ tends to $\infty$. Then as before, elliptic theory ensures that $H$ is smooth and

$$
i \Lambda F_{H}=\lim _{i \rightarrow \infty} i \Lambda F_{H_{l}}=0,
$$

i.e., the Chern connection of $H$ is an Hermitian Yang-Mills connection on $\left.\mathcal{E}\right|_{Y \times R}$ with respect to the tube metric $(g+d t \otimes d t)$. This proves (1) of Theorem 2.1. 
By the construction of $H$, it also follows that

$$
d\left(H, H_{0}^{\prime}\right)=\lim _{i \rightarrow \infty} d\left(H_{i}, H_{0}^{\prime}\right) \leqq C
$$

and

$$
d\left(H, H_{0}^{\prime}\right)(x, t)=O\left(e^{-\delta_{\lambda} t}\right), \quad t \longrightarrow \infty
$$

The fact

$$
d\left(H, H_{0}^{\prime}\right)(x, t)=O\left(e^{-\delta_{\lambda} t}\right), \quad t \longrightarrow \infty
$$

shows that $H$ extends continuously over $\Sigma_{\infty}$ on which it is the same as $H_{0}^{\prime}$, i.e. the given unitary structure of $\mathcal{E}$ over $\Sigma_{\infty}$. Thus (2) of Theorem 2.1 is also proved.

We now go on to show (3) of Theorem 2.1. We need the following lemma.

Lemma 5.4. For the solution $H$ obtained above, we have the following estimates on its derivatives:

$$
\begin{aligned}
\left|A_{H}-A_{H_{0}^{\prime}}\right|=o(1), \quad t \rightarrow \infty, \\
\left|d_{A_{H_{0}^{\prime}}}\left(A_{H_{0}^{\prime}}-A_{H}\right)\right|=o(1), \quad t \rightarrow \infty .
\end{aligned}
$$

The lemma is a direct consequence of the proposition in the appendix. The proof of the proposition involves an application of the Schauder estimates and a scaling argument.

(3) now follows easily from Lemma 5.4, for by Lemma 5.4, $A_{H}$ and $A_{H_{0}^{\prime}}$ must have the same asymptotic limits as $t \rightarrow \infty$ and clearly $A_{H_{0}^{\prime}}$ limits to the lift to $Y$ of the flat stucture of $\mathcal{E}$ over $\Sigma_{\infty}$. This completes the proof of Theorem 2.1 .

With Theorem 2.1 in hand we can now describe the map from $\mathcal{M}_{c}^{*}(+,+)$ to the set of gauge equivalence classes of ASD connections on $Y \times R$.

Let $\mathcal{E} \in \mathcal{E}_{c}(+,+)$ be a holomorphic bundle on $S$ representing an element of $\mathcal{M}_{c u}^{*}(+,+)$ and let $H$ be the unique Hermitian metric on $\mathcal{E}$ as in Theorem 2.1. Note that the smooth bundle underlying $\left.\mathcal{E}\right|_{Y \times R}$ is necessarily isomorphic to the bundle $E$ as smooth bundles, thus we can identify the former with $E$ and consider $\left.\mathcal{E}\right|_{Y \times R}$ as a holomorphic structure on $E$. The connection $A_{H}$ then is an ASD connection on $E$ over $Y \times R$. In general it is not compatible with the metric $H_{0}$. However, we can associate with it an ASD connection $A_{H_{0}}$ on $\left(E, H_{0}\right)$ in a canonical way as follows.

Pull $A_{H}$ back to a connection $A_{H_{0}}$ on $\left(E, H_{0}\right)$ by the positive self-adjoint gauge transformation $g$ of $(E, H)$ (as well as $\left(E, H_{0}\right)$ ) determined by

$$
\langle\eta, \xi\rangle_{H_{0}}=\langle g \eta, g \xi\rangle_{H}
$$

The connection $A_{H_{0}}$ plainly has the same energy (finite or infinite) and asymptotic limits (exist or not) as those of $A_{H}$. Furthermore the uniqueness of Theorem 2.1 implies that the gauge equivalence class of ASD connections on $\left(E, H_{0}\right)$ represented by $A_{H_{0}}$ depends only on the equivalence class of $\mathcal{M}_{c u}(+,+)$ represented by $\mathcal{E}$, not on $\mathcal{E}$ itself. So there is a map from $\mathcal{M}_{c u}(+,+)$ to the set of gauge equivalence classes of ASD connections on $Y \times R$. By the discussion following the definitions of $\mathcal{M}_{c}(+,+)$ and $\mathcal{M}_{c u}(+,+)$, we see that this also gives a map from $\mathcal{M}_{c}^{*}(+,+)$ to the set of gauge equivalence classes of ASD connections on $Y \times R$. 


\section{From Instantons on $Y \times R$ to Holomorphic Bundles on $S$}

In this section, we prove Theorem 2.2.

Let $\left(E, H_{0}\right)$ be a fixed complex Hermitian bundle associated with the principal $S U(2)$ bundle $P=Y \times R \times S U(2)$. We identify connections on $P$ with connections on $E$ compatible with $H_{0}$. In this section, connections on $E$ will always be taken to be compatible with the hermitian metric on $H_{0}$ unless otherwise stated.

Given an anti-self-dual connection $A$ on $E$ with finite energy, it is well-known that $A$ determines a holomorphic structure on $E$, viewed as a complex vector bundle on the complex manifold $Y \times R \subset S$. On the other hand, we have seen in Sect. 1 that the one parameter family of connections $\left[A_{t}\right]$ on $Y$ obtained in an obvious way from $A$ converges in $C^{\infty}$ to a flat connection $\left[A_{\infty}\right]$ (resp. $\left.\left[A_{-\infty}\right]\right)$ as $t \rightarrow \infty($ resp. $-\infty)$ and if $\left[A_{ \pm \infty}\right] \in \mathcal{F}_{+}^{*}$, then we can choose a gauge for $E$ such that the connection form and its covariant derivatives decay exponentially at the ends. Rewriting this in terms of complex coordinates on $S$, we shall see that these are sufficient conditions to extend the bundle $E$ on $Y \times R$ over the whole $S$.

Theorem 6.1. Let $A$ be an anti-self-dual connection on $E$ with finite energy. If $\left[A_{ \pm \infty}\right] \in \mathcal{F}_{+}^{*}$, then the following three statements hold.

(1) The bundle $E$ on $Y \times R$ with the holomorphic structure determined by $A$ extends to a holomorphic bundle $\mathcal{E}$ on $S$ such that

$$
c_{2}(\mathcal{E})=\frac{1}{8 \pi^{2}}\left\|F_{A}\right\|_{L^{2}}^{2}
$$

(2) The restrictions of the bundle $\mathcal{E}$ over $\Sigma_{0}$ and $\Sigma_{\infty}$ are unitary flat. The flat structure on $\Sigma_{0}$, when lifted to $Y$, is given by $\left[A_{-\infty}\right]$ and the flat structure on $\Sigma_{\infty}$, when lifted to $Y$, is given by $\left[A_{\infty}\right]$.

(3) The Hermitian metric $H_{0}$ on E extends continuously over $\Sigma_{0}$ and $\Sigma_{\infty}$ to a Hermitian metric on $\mathcal{E}$. The extended metric agrees with the unitary structures of the restrictions of $\mathcal{E}$ on $\Sigma_{0}$ and $\Sigma_{\infty}$ given in (2).

We prove Theorem 6.1 in a series of lemmas. Roughly speaking, we first extend $E$ and $A$ locally by solving a local " $\bar{\partial} "$ equation. We then show that the local extensions patch together to give a global extension. Finally we show that the extended bundle satisfies (2) and (3). We shall only prove the theorem for the end $\Sigma_{\infty}$. The proof for $\Sigma_{0}$ is almost identical.

Recall that $Y \times R$ is the complement to the zero section of the line bundle $L$. As in Sect. 3, let $\left(w, z_{h}\right)$ be a local complex coordinate on $L$ coming from some holomorphic trivialization of $L$. Let $z=\frac{1}{z_{h}}$, then $z=0$ represent points of $\Sigma_{\infty}$ in $S$ and $(w, z)$ is a local complex coordinate in $S$ around these points. We can take such a complex coordinate around any point of $S$ in $\Sigma_{\infty}$. So without loss of anything we suppose $U$ is a neighbourhood in $S$ of some point in $\Sigma_{\infty}$ with $(w, z)$ defined on it. Clearly $U$ contains a subset of $Y \times R$ of the form $\left.Y\right|_{W} \times[T, \infty)$, where $W$ is some open subset of $\Sigma$ on which $w$ is defined and $T$ is some positive number. So $E$ and $A$ are defined on $U \backslash\{z=0\}$. We first extend $E$ over $\{z=0\}$ locally on each such $U$.

For convenience of presentation, we denote $e_{1}=d z, e_{2}=d \bar{z}, e_{3}=d w$ and $e_{4}=d \bar{w}$, and we define $\sigma\left(i_{1}, \ldots, i_{j}\right)$, for any $j$-tuple $\left(i_{1}, \ldots, i_{j}\right)$ of integers with $1 \leqq i_{l} \leqq 4$, to be the total number of 1 and 2 appearing in $i_{1}, \ldots, i_{j}$, for example 
$\sigma(1,2,4,1)=3$. Note that by the formula of $\Omega_{0}$ on the cotangent bundle $T^{*}(U \backslash\{z=$ $0\})$ in Sect. 3, we have $\left|e_{1}\right|_{\Omega_{0}},\left|e_{2}\right|_{\Omega_{0}}=O(|z|),\left|e_{3}\right|_{\Omega_{0}},\left|e_{4}\right|_{\Omega_{0}}=O(1)$.

Lemma 6.2. For $U$ small enough, we can choose a gauge $g_{U}$ on $\left.E\right|_{U \backslash\{z=0\}}$ such that the connection form $A$ satisfies the following condition: if

Identify $\operatorname{End}\left(\left.E\right|_{U \backslash\{z=0\}}\right)$ with the trivial bundle and $A$ as a section of it by $g_{U}$,

$$
\nabla^{j} A=\sum_{1 \leqq i_{0} \cdots i_{j} \leqq 4} A_{i_{0} \cdots i_{j}} e_{i_{0}} \otimes e_{i_{1}} \otimes \cdots \otimes e_{i_{j}}, \quad j=0,1,2, \ldots,
$$

where $\nabla$ is the covariant derivative of the trivial connection on $E n d\left(\left.E\right|_{U \backslash\{z=0\}}\right) \otimes$ $T^{*}(U \backslash\{z=0\})$, then

$$
\left|A_{i_{0} \cdots i_{j}}\right|=O\left(\frac{1}{|z|^{\sigma\left(i_{0} \cdots i_{j}\right)-\delta}}\right)
$$

for some positive number $\delta$.

Proof. We prove the following two claims. The result then follows.

Claim A) If $B=B_{l_{1} \cdots l_{k}} e_{l_{1}} \otimes \cdots \otimes e_{l_{k}}$ is a $k$ covariant tensor on $U \backslash\{z=0\}$ such that

$$
|B|_{\Omega_{0}}=O\left(\frac{1}{|z|^{-\delta}}\right), \quad|z| \rightarrow 0,
$$

then

$$
\left|B_{l_{1} \cdots l_{k}}\right|=O\left(\frac{1}{|z|^{\sigma\left(l_{1} \cdots l_{k}\right)-\delta}}\right) .
$$

Claim B) There is a gauge $g_{U}$ on $\left.E\right|_{U \backslash\{z=0\}}$ such that the connection form $A$ of the instanton satisfies

$$
\left|\nabla^{j} A\right|_{\Omega_{0}}=O\left(\frac{1}{|z|^{-\delta}}\right), \quad j=0,1,2, \ldots .
$$

Claim A) follows easily from the formula of $\Omega_{0}$ on the cotangent bundle in Lemma 3.2.

To prove Claim B), we note that by the discussion in Sect. 1, we can choose a gauge on $E$ such that

$$
\left|\nabla_{A_{\infty}}^{j}\left(A-A_{\infty}\right)\right|_{\Omega_{0}} \leqq C e^{-\delta t}, \quad t \longrightarrow \infty, \quad j=0,1,2, \ldots .
$$

Here the covariant derivative $\nabla_{A_{\infty}}$ on $\operatorname{End}(E) \otimes T^{*}(U \backslash\{z=0\})$ is induced by the connection $A_{\infty}$ on $\left.E\right|_{U \backslash\{z=0\}}$ and the Levi-Civita connection of the metric $\Omega_{0}$ on $T^{*}(U \backslash\{z=0\})$, hence it is different from the covariant derivative $\nabla$ required in Claim B).

Since $A_{\infty} \in F_{+}$, it is trivial on $U$ if $U$ is small enough. Thus, if neccessary shrink $U$ to a smaller open subset, we can choose a local gauge $g_{U}$ in which $A_{\infty}=0$ locally. So we have

$$
\left|\nabla_{A_{\infty}}^{j} A\right|_{\Omega_{0}} \leqq C e^{-\delta t}, \quad j=0,1, \ldots
$$

i.e.

$$
(\star \star) \quad\left|\nabla_{A_{\infty}}^{j} A\right|_{\Omega_{0}}=O\left(\frac{1}{|z|^{-\delta}}\right), \quad j=0,1,2, \ldots
$$


To finish the proof, we induct on $j$. It is trivial for $j=0$. Suppose $\left|\nabla^{k} A\right|_{\Omega_{0}}=$ $O\left(\frac{1}{|z|^{-\delta}}\right)$ and let

$$
\nabla^{k} A=A_{i_{0} \cdots i_{k}} e_{i_{0}} \otimes \cdots \otimes e_{i_{k}},
$$

then by Claim A),

$$
(\star \star \star)\left|A_{i_{0} \cdots i_{k}}\right|=O\left(\frac{1}{|z|^{\sigma\left(i_{0} \cdots i_{k}\right)-\delta}}\right) .
$$

Since $A_{\infty}=0$, we see that

$$
\nabla_{A_{\infty}}^{k+1} A=\nabla^{k+1} A+A_{i_{0} \cdots i_{k}} \nabla_{\Omega_{0}}\left(e_{i_{0}} \otimes \cdots \otimes e_{i_{k}}\right),
$$

where $\nabla_{\Omega_{0}}$ is the covariant derivative of the Levi-Civita connection of the metric $\Omega_{0}$ on tensor bundles of $U \backslash\{z=0\}$. By ( $\left.\star \star\right)$, it suffices to show that

$$
\left|A_{i_{0} \cdots i_{k}} \nabla_{\Omega_{0}}\left(e_{i_{0}} \otimes \cdots \otimes e_{i_{k}}\right)\right|_{\Omega_{0}}=O\left(\frac{1}{|z|^{-\delta}}\right) .
$$

Working out the connection form of $\nabla_{\Omega_{0}}$ under the basis $\left\{e_{1}, e_{2}, e_{3}, e_{4}\right\}$ (i.e. the Cristoffel coefficients. This is of course a clumsy way, but the author knows no simpler way of reasoning), we see that

$$
\left|\nabla_{\Omega_{0}} e_{1}\right|_{\Omega_{0}},\left|\nabla_{\Omega_{0}} e_{2}\right|_{\Omega_{0}}=O(|z|),
$$

and

$$
\left|\nabla_{\Omega_{0}} e_{3}\right|_{\Omega_{0}},\left|\nabla_{\Omega_{0}} e_{4}\right|_{\Omega_{0}}=O(1)
$$

It follows that

$$
\left|\nabla_{\Omega_{0}}\left(e_{i_{0}} \otimes \cdots \otimes e_{i_{k}}\right)\right|_{\Omega_{0}}=O\left(|z|^{\sigma\left(i_{0} \cdots i_{k}\right)}\right),
$$

which together with $(\star \star \star)$ imply that

$$
\left|A_{i_{0} \cdots i_{k}} \nabla_{\Omega_{0}}\left(e_{i_{0}} \otimes \cdots \otimes e_{i_{k}}\right)\right|_{\Omega_{0}}=O\left(\frac{1}{|z|^{-\delta}}\right) .
$$

This finishes the proof of Lemma 6.2.

The following Lemma is due to Buchdahl [4]. It is the key lemma in our local extension of $E$.

Lemma 6.3. Let $U$ be a neighbourhood of the origin in $C^{2}$. Let $A \in L_{j}^{p}(U)$, $j \geqq 1, p \geqq 4$ be a matrix valued $(0,1)$-form on $U$ such that

$$
\bar{\partial} A+A \wedge A=0,
$$

then there is a matrix function $g \in L_{j+1}^{p}(U)$, possibly defined on a smaller neighbourhood, such that

$$
\bar{\partial} g=-A g \text {. }
$$


Proof. See Lemma 8 in Buchdahl's paper [4].

To extend $E$ locally on $U \backslash\{z=0\}$ over $\{z=0\}$, we consider, following Bando [2], a branched covering $U^{m}$ of $\mathrm{U}$ :

$$
\begin{gathered}
\phi: U^{m} \longrightarrow U, \\
\left(w^{\prime}, z^{\prime}\right) \mapsto(w, z)=\left(w^{\prime}, z^{\prime m}\right),
\end{gathered}
$$

where $m$ is some positive integer. Pull back $E$ and $A$ on $U \backslash\{z=0\}$ to $U^{m} \backslash\left\{z^{\prime}=0\right\}$ by $\phi$. Denote the pull-back bundle and connection still by $E$ and $A$. The identity of $E$ is pulled back to a cyclic transformation group $G_{m}$ of order $m$ of the pull-back bundle on $U^{m}$ and the gauge $g_{U}$ to an $G_{m}$ invariant gauge, which will be denoted still by $g_{U}$, for the pull-back bundle. Clearly $G_{m}$ acts holomorphically. By simple calculation, one sees that Lemma 6.2 implies that on $\left(w^{\prime}, z^{\prime}\right)$ plane,

$$
\left|\nabla^{j} A\right|=O\left(\frac{1}{\left|z^{\prime}\right|^{(j+1-m \delta)}}\right) .
$$

Thus for any given $j$, we can choose $m$ large enough so that

$$
A \in L_{j}^{p}\left(U^{m}\right),
$$

and in particular we can suppose that $A$ vanishes on $z^{\prime}=0$. On the other hand, the anti-self-duality of $A$ means that on both $U$ and $U^{m}$ planes $A$ satisfies

$$
\bar{\partial} A^{0,1}+A^{0,1} \wedge A^{0,1}=0 .
$$

So by Lemma 6.3 , shrink $U^{m}$ if neccessary, there is a matrix function $g \in L_{j+1}^{p}\left(U^{m}\right)$ such that

$$
\bar{\partial} g=-A^{0,1} g .
$$

Note that there are many functions satisfying the above equation, for if $g$ is such a one, so is $g f$ for any holomorphic matrix function $f$ on $U^{m}$. Since $A$ vanishes on $z^{\prime}=0$, we see that $g(0, w)$ is holomorphic; thus we suppose $g$ is the identity over $z^{\prime}=0$, for if neccessary, we can always take $g \cdot g^{-1}(0, w)$ instead.

Now let $g_{U}^{\prime}=g_{U} g$, under $g_{U}^{\prime}$ the connection form is given by

$$
g^{-1} A g+g^{-1} d g=g^{-1} A^{(1,0)} g+g^{-1} \partial g,
$$

i.e. it is of type $(1,0)$. Thus $g_{U}^{\prime}$ is a holomorphic basis of the pull-back bundle on $U^{m} \backslash\left\{z^{\prime}=0\right\}$. We extend the pull-back bundle holomorphically over $\left\{z^{\prime}=0\right\}$ by defining $g_{U}^{\prime}$ as a holomorphic basis on $U^{m}$.

Consider now the action of $G_{m}$ on $g_{U}^{\prime}$. Denote by $t_{0}$ the generator of $G_{m}$ which covers the multiplication by $e^{\frac{2 \pi u}{m}}$ on the $z^{\prime}$ coordinate on $U^{m}$ downstair. Since $g_{U}$ is invariant under $G_{m}$, we have

$$
\begin{aligned}
\left(t_{0} g_{U}^{\prime}\right)\left(e^{\frac{2 \pi u}{m}} z^{\prime}, w\right) & =\left(t_{0} g_{U}\right)\left(e^{\frac{2 \pi u}{m}} z^{\prime}, w\right) g\left(z^{\prime}, w\right)=g_{U}\left(e^{\frac{2 \pi u}{m}} z^{\prime}, w\right) g\left(z^{\prime}, w\right) \\
& =g_{U}^{\prime}\left(e^{\frac{2 \pi l}{m}} z^{\prime}, w\right) g^{-1}\left(e^{\frac{2 \pi i}{m}} z^{\prime}, w\right) g\left(z^{\prime}, w\right) .
\end{aligned}
$$

As both the action and the basis $g_{U}^{\prime}$ are holomorphic, the matrix representation of the action under the basis, that is $g^{-1}\left(e^{\frac{2 \pi t}{m}} z^{\prime}, w\right) g\left(z^{\prime}, w\right)$, is also holomorphic. On the other hand, $g$ is differentiable on $U^{m}$, being in $L_{j+1}^{p}\left(U^{m}\right)$ for a given $j$ large enough, 
one sees that $g^{-1}\left(e^{\frac{2 \pi u}{m}} z^{\prime}, w\right) g\left(z^{\prime}, w\right)$ is bounded, hence it extends holomorphically over $z^{\prime}=0$, on which it is easily seen to be the identity matrix $I$. Consequently $G_{m}$ extends to a holomorphic transformation group on the extended bundle which acts as identity over $\left\{z^{\prime}=0\right\}$. It follows that

$$
g_{U}^{\prime \prime}=\sum_{t \in G_{m}} t\left(g_{U}^{\prime}\right)
$$

is a $G_{m}$-invariant holomorphic basis of the extended bundle on $U^{m}$ or perhaps a smaller open subset. So the quotient of the extended bundle by $G_{m}$ is a holomorphic bundle on $U$ which clearly is a holomorphic extension of the bundle $\left.E\right|_{U \backslash\{z=0\}}$ and $g_{U}^{\prime \prime}$ projects to a holomorphic basis, which we still denote by $g_{U}^{\prime \prime}$, of this extension of $E$ on $U$.

We now look at $g^{\prime \prime}$ more closely. By construction

$$
\begin{aligned}
g^{\prime \prime}\left(z^{\prime}, w\right) & =g_{U}^{\prime}\left(z^{\prime}, w\right)\left[I+g^{-1}\left(z^{\prime}, w\right) g\left(e^{\frac{-2 \pi i}{m}} z^{\prime}, w\right)+\cdots+g^{-1}\left(z^{\prime}, w\right) g\left(e^{\frac{-(m-1) 2 \pi t}{m}} z^{\prime}, w\right)\right] \\
& =g_{U}\left(z^{\prime}, w\right)\left[g\left(z^{\prime}, w\right)+g\left(e^{\frac{-2 \pi i}{m}} z^{\prime}, w\right)+\cdots+g\left(e^{\frac{-(m-1) 2 \pi t}{m}} z^{\prime}, w\right)\right] .
\end{aligned}
$$

Let $T_{U}\left(z^{\prime}, w\right)=g\left(z^{\prime}, w\right)+g\left(e^{\frac{-2 \pi i}{m}} z^{\prime}, w\right)+\cdots+g\left(e^{\frac{-(m-1) 2 \pi u}{m}} z^{\prime}, w\right)$. Since both $g_{U}^{\prime \prime}$ and $g_{U}$ are $G_{m}$ invariant, $T_{U}\left(z^{\prime}, w\right)$ is a $G_{m}$ invariant matrix function, as can be seen directly. Thus it descends to a matrix function on $U \backslash\{z=0\}$ which we still denote by $T_{U}$. Clearly on the $(z, w)$ plane,

$$
g^{\prime \prime}(z, w)=g(z, w) T_{U}(z, w) .
$$

Since $g\left(z^{\prime}, w\right) \rightarrow I$, as $z^{\prime} \rightarrow 0$, we see that $T_{U}(z, w) \rightarrow m I$ as $z \rightarrow 0$, thus it has a continuous extension over $\{z=0\}$, on which it is the constant matrix $m I$. Consequently, $g_{U}$ can also be extended over $\{z=0\}$ at least continuously to give a basis of the extended bundle on $U$ and in turn the Hermitian metric $H_{0}$ on $\left.E\right|_{U \backslash\{z=0\}}$ can be extended over $z=0$ to give a Hermitian metric on the extended bundle which is continuous and smooth away from $z=0$ and in the $w$ direction at $z=0$.

Up to now, we have showed that locally $\left.E\right|_{S \backslash \Sigma_{\infty}}$ extends over $\Sigma_{\infty}$. We now show that these local extensions, patch together to give a global holomorphic extension of $E$ over $\Sigma_{\infty}$. It suffices to show that for any $U_{1}$ and $U_{2}$ as above, the transition function of the two gauges $g_{U_{1}}^{\prime \prime}$ and $g_{U_{2}}^{\prime \prime}$, which is defined and holomorphic on $U_{1} \cap U_{2} \backslash \Sigma_{\infty}$, extends to a holomorphic function on $U_{1} \cap U_{2}$, for then the cocycle condition follows from the continuity of the transition functions and the cocycle condition for $\left.E\right|_{S \backslash \Sigma_{\infty}}$.

Let $g_{U_{1}}=g_{U_{2}} T$. So $T$ is a function on $U_{1} \cap U_{2} \backslash \Sigma_{\infty}$ with value in $S U(2)$. In fact $T$ is constant because the connection forms of the flat connection $A_{\infty}$ under $g_{U_{1}}$ and $g_{U_{2}}$ vanish. So in particular it is bounded. The transition of $g_{U_{1}}^{\prime \prime}$ and $g_{U_{2}}^{\prime \prime}$ is

$$
g_{U_{1}}^{\prime \prime}=g_{U_{1}} T_{U_{1}}=g_{U_{2}} T T_{U_{1}}=g_{U_{2}}^{\prime \prime} T_{U_{2}}^{-1} T T_{U_{1}} .
$$

Since $T_{U_{1}}$ and $T_{U_{2}}$ are bounded, being continuous, the matrix function $T_{U_{2}}^{-1} T T_{U_{1}}$, which is holomorphic on $U_{1} \cap U_{2} \backslash \Sigma_{\infty}$, is also bounded on $U_{1} \cap U_{2} \backslash \Sigma_{\infty}$, hence it extends holomorphically over $U_{1} \cap U_{2} \cap \Sigma_{\infty}$. This proves the first half of (1) of Theorem 6.1.

Next we show that the extended bundle is unitary flat over $\Sigma_{\infty}$ with the flat structure which, when lifted to over $Y$, is given by $\left[A_{\infty}\right]$. This is quite obvious 
from the above discussion, for at $z=0, g_{U}^{\prime \prime}(0, w)$ 's give a trivialization of the restriction of the extended bundle. The transition between $g_{U_{1}}^{\prime \prime}(0, w)$ and $g_{U_{2}}^{\prime \prime}(0, w)$ is $T_{U_{2}}^{-1} T T_{U_{1}}(0, w)$, i.e. $T(0, w)$. As mentioned above, $T$ is constant, thus $g_{U}^{\prime \prime}(0, w)$ 's give a flat structure of the restriction of the extended bundle over $z=0$. Since $T$ takes value in $S U(2)$, being the transition function between two gauges of an $S U(2)$ bundle, the flat structure is also a special unitary flat structure. Since $g_{U}^{\prime \prime}(0, w)$ 's are holomorphic gauges, the flat structures is compatible with the holomorphic structure.

Now take $Y$ to be the boundary of a tubular neighbourhood of $\Sigma_{\infty}$, small enough to be covered by the $U$ 's in the above extension. (Of course the orientation of $Y$ is reversed, but it does not matter here.), Then the local gauges $\left.g_{U}\right|_{Y}$ 's determine the flat structure $\left[A_{\infty}\right]$. Since $\left.g_{U}\right|_{Y}$ 's have the same transition functions as $g_{U}^{\prime \prime}(0, w)$ 's, we see that $\left[A_{\infty}\right]$ is the lift of the new unitary flat structure over $\Sigma_{\infty}$. This proves (2) of Theorem 6.1.

Since each of the original unitary gauges $g_{U}$ also extends over $\Sigma_{\infty}$ to a local frame of the extended bundle, the Hermitian metric $H_{0}$ on $E$ extends at least continuously over $\Sigma_{\infty}$ to give a Hermitian metric on $\mathcal{E}$ which, restricted to $\Sigma_{\infty}$, is clearly the same unitary structure of $\mathcal{E}$ on $\Sigma_{\infty}$ defined by the transition $T$ above. This shows (3).

Finally, to complete the proof of Theorem 6.1, we show that the second Chern number of the bundle $\mathcal{E}$ on $S$ thus obtained equals $\frac{1}{8 \pi^{2}}\left\|F_{A}\right\|_{L^{2}}^{2}$. Since $\mathcal{E}$ is an element of $\mathcal{E}_{c}(+,+)$, Lemma 5.2 implies that there is a smooth metric $H_{0}^{\prime}$ on $\mathcal{E}$ which extends the unitary structures over $\Sigma_{0}$ and $\Sigma_{\infty}$, i.e. agrees with $H_{0}$ on the ends. On the other hand, by uniqueness the solution $H$ in Theorem 2.1 for $\mathcal{E}$ must be the Hermitian metric $H_{0}$ we start with. So in this case, (1) of the proposition of the appendix is satisfied for both ends $\Sigma_{0}$ and $\Sigma_{\infty}$. Consequently we have the following estimates:

$$
\left|A_{H_{0}^{\prime}}-A_{H_{0}}\right|=o(1), \quad t \rightarrow \pm \infty,
$$

and

$$
\left|d_{A_{H_{0}^{\prime}}}\left(A_{H_{0}^{\prime}}-A_{H_{0}}\right)\right|=o(1), \quad t \rightarrow \pm \infty .
$$

On the other hand, by the Chern-Weil formula, we have

$$
\begin{aligned}
\int_{D_{i j}} \operatorname{Tr}\left(F_{H_{0}^{\prime}} \wedge F_{H_{0}^{\prime}}\right)-\int_{D_{i j}} \operatorname{Tr}\left(F_{H_{0}} \wedge F_{H_{0}}\right) \\
\quad=\int_{\partial D_{\imath \jmath}} \operatorname{Tr}\left\{\left(A_{H_{0}^{\prime}}-A_{H_{0}}\right) \wedge\left[2 F_{H_{0}^{\prime}}+d_{A_{H_{0}^{\prime}}}\left(A_{H_{0}^{\prime}}-A_{H_{0}}\right)+\frac{2}{3}\left(A_{H_{0}^{\prime}}-A_{H_{0}}\right) \wedge\left(A_{H_{0}^{\prime}}-A_{H_{0}}\right)\right]\right\} .
\end{aligned}
$$

It follows that

$$
\begin{aligned}
c_{2}(\mathcal{E})= & \frac{1}{8 \pi^{2}} \int_{S} \operatorname{Tr}\left(F_{H_{0}^{\prime}} \wedge F_{H_{0}^{\prime}}\right)=\lim _{i, j \rightarrow \infty} \frac{1}{8 \pi^{2}} \int_{D_{\imath \jmath}} \operatorname{Tr}\left(F_{H_{0}^{\prime}} \wedge F_{H_{0}^{\prime}}\right) \\
= & \lim _{i, j \rightarrow \infty} \frac{1}{8 \pi^{2}}\left\{\int_{D_{i j}} \operatorname{Tr}\left(F_{H_{0}} \wedge F_{H_{0}}\right)+\int_{\partial D_{i j}} \operatorname{Tr}\left[\left(A_{H_{0}^{\prime}}-A_{H_{0}}\right)\right.\right. \\
& \left.\left.\wedge\left(2 F_{H_{0}^{\prime}}+d_{A_{H_{0}^{\prime}}}\left(A_{H_{0}^{\prime}}-A_{H_{0}}\right)+\frac{2}{3}\left(A_{H_{0}^{\prime}}-A_{H_{0}}\right) \wedge\left(A_{H_{0}^{\prime}}-A_{H_{0}}\right)\right)\right]\right\} \\
= & \frac{1}{8 \pi^{2}} \int_{Y \times R} \operatorname{Tr}\left(F_{H_{0}} \wedge F_{H_{0}}\right)+0=\frac{1}{8 \pi^{2}}\left\|F_{A}\right\|_{L^{2}}^{2} .
\end{aligned}
$$

This completes the proof of Theorem 6.1. 
Theorem 6.1 essentially gives the map of Theorem 2.2. We now describe this in precise terms. We need two small lemmas to put things straight.

Lemma 6.4. Let $U$ be a local coordinate neighbourhood of some point of $\Sigma_{\infty}$ as above. Let $A_{0}$ and $A_{1}$ be two 1-forms on $U \backslash \Sigma_{\infty}$ with values in the Lie algebra su(2) of $S U(2)$ and $g_{U}$ be a function on $U \backslash \Sigma_{\infty}$ with values in $S U(2)$. Suppose the three are related by

$$
A_{0}=g_{U}^{-1} A_{1} g_{U}+g_{U}^{-1} d g_{U}
$$

If there is a real number $\delta>0$ such that for $k \geqq 0$,

$$
\begin{aligned}
& \left|\nabla^{k} A_{0}\right|_{\Omega_{0}}=O\left(e^{-\delta t}\right), \quad t \rightarrow \infty, \\
& \left|\nabla^{k} A_{1}\right|_{\Omega_{0}}=O\left(e^{-\delta t}\right), \quad t \rightarrow \infty,
\end{aligned}
$$

where $\nabla$ denote the covariant differentiation of the trivial connection on $U \backslash \Sigma_{\infty} \times$ $s u(2)$ tensored with the Levi-Civita connection of the tube metric on forms, then $g_{U}(y, t)$ converges to some constant $S U(2)$ valued function on $\left.Y\right|_{U \cap \Sigma_{\infty}}$, hence also on $U \cap \Sigma_{\infty}$.

Proof. This is simple. We write

$$
d g_{U}=A_{0} g_{U}-g_{U} A_{1} .
$$

Since $g_{U}$ is $S U(2)$ valued, it is bounded. By the hypothesis

$$
\left|d g_{U}\right|_{\Omega_{0}} \leqq C\left(\left|A_{0}\right|_{\Omega_{0}}+\left|A_{1}\right|_{\Omega_{1}}\right) \leqq C e^{-\delta t}
$$

Here and in the following $C$ denote some general constant large enough. In particular

$$
\left|\frac{\partial g_{U}}{d t}\right| \leqq C\left(e^{-\delta t}\right)
$$

and

$$
\left|d_{Y} g_{U}\right| \leqq C e^{-\delta t}
$$

The result then follows easily.

In Theorem 6.1, we restrict ourselves to instantons on $E$ compatible with $H_{0}$. Of course the construction applies to instantons compatible with other Hermitian metrics on $E$ as well.

Lemma 6.5. Let $A_{0}$ be an instanton on $\left(E, H_{0}\right)$ and $A_{1}$ be another one on $\left(E, H_{1}\right)$ for some other Hermitian metric $H_{1}$ on $E$. If $A_{0}$ and $A_{1}$ are gauge equivalent by a gauge $g \in \operatorname{Aut}(E)$, then the holomorphic bundles $\mathcal{E}_{0}$ and $\mathcal{E}_{1}$ on $S$, constructed from $A_{0}$ and $A_{1}$ in Theorem 6.1, are isomorphic as bundles in $\mathcal{E}_{c}(+,+)$ by an isomorphism which extends $g$ and is unitary with respect to the unitary structures of $\mathcal{E}_{0}$ and $\mathcal{E}_{1}$ over the ends $\Sigma_{0}$ and $\Sigma_{\infty}$ constructed in Theorem 6.1. 
Proof. Note that the gauge transformation $g$ is a holomorphic bundle isomorphism between $\left.\mathcal{E}_{0}\right|_{Y \times R}$ and $\left.\mathcal{E}_{1}\right|_{Y \times R}$. We shall show that $g$ extends locally over $\{z=0\}$ on each local coordinate $U$ as above to give a holomorphic isomorphism between $\left.\mathcal{E}_{0}\right|_{U}$ and $\left.\mathcal{E}_{1}\right|_{U}$. The extension is also unitary on $\{z=0\}$. Then by the continuity of the extensions, these local extensions of $g$ necessarily agree on overlaps, hence gives a global holomorphic extension of $g$. The extension is by construction unitary on $\Sigma_{\infty}$. Thus $\mathcal{E}_{0}$ and $\mathcal{E}_{1}$ are isomorphic as bundles in $\mathcal{E}_{c}(+,+)$.

To show that $g$ extends locally on $U$, recall that in the construction of $\mathcal{E}_{0}$ and $\mathcal{E}_{1}$, there is an $S U(2)$ gauge $g_{0}$ on $\left.\left(E, H_{0}\right)\right|_{U \backslash \Sigma_{\infty}}$ and an $S U(2)$ gauge $g_{1}$ on $\left.\left(E, H_{1}\right)\right|_{U \backslash \Sigma_{\infty}}$ such that the connection form of $A_{0}$ under $g_{0}$ satisfies

$$
\left|\nabla^{k} A_{0}\right|_{\Omega_{0}}=O\left(e^{-\delta_{0} t}\right), \quad t \rightarrow \infty
$$

for some real number $\delta_{0}>0$, and the connection form of $A_{1}$ under $g_{1}$ satisfies

$$
\left|\nabla^{k} A_{1}\right|_{\Omega_{1}}=O\left(e^{-\delta_{1} t}\right), \quad t \rightarrow \infty
$$

for some real number $\delta_{1}>0$. Recall also that there is a holomorphic gauge $g_{0}^{\prime \prime}$ of $\left.\mathcal{E}_{0}\right|_{U}$ such that

$$
g_{0}^{\prime \prime}=g_{0} T_{0}(z, w)
$$

for some $G L(2, C)$ valued function $T_{0}(z, w)$ such that $T_{0}(z, w) \rightarrow m I$ as $z \rightarrow 0$, and there is a holomorphic gauge $g_{1}^{\prime \prime}$ of $\left.\mathcal{E}_{1}\right|_{U}$ such that

$$
g_{1}^{\prime \prime}=g_{1} T_{1}(z, w)
$$

for some $G L(2, C)$ valued function $T_{1}(z, w)$ such that $T_{1}(z, w) \rightarrow m I$ as $z \rightarrow 0$. Now let $g_{U}$ be the matrix of the gauge transformation $g$ under the basis $g_{0}$ and $g_{1}$, i.e.,

$$
g\left(g_{0}\right)=g_{1} g_{U}
$$

$g$ being a gauge transformation between $\left(E, H_{0}\right)$ and $\left(E, H_{1}\right)$, we see that $g_{U}$ is $S U(2)$ valued. Since $g$ pulls $A_{1}$ back to $A_{0}$, we have

$$
g_{U}^{-1} A_{1} g_{U}+g_{U}^{-1} d g_{U}^{-1}=A_{0}
$$

i.e.,

$$
g_{U}^{-1} d g_{U}=A_{0}-g_{U}^{-1} A_{1} g_{U} .
$$

It follows from Lemma 6.4 that

$$
g_{U} \rightarrow g_{\infty}
$$

as $t \rightarrow \infty$, or equivalently $z \rightarrow 0$ on $U$, for some $S U(2)$ valued function $g_{\infty}$ on $\Sigma_{\infty}$ which is actually constant. Now consider the transformation $g$ under the holomorphic gauges $g_{0}^{\prime \prime}$ and $g_{1}^{\prime \prime}$,

$$
g\left(g_{0}^{\prime \prime}\right)=g_{1}^{\prime \prime} T_{1}^{-1} g_{U} T_{0} .
$$

$T_{1}^{\prime-1} g_{U} T_{0}$ is defined and holomorphic on $U \backslash \Sigma_{\text {infty }}$, for $g$ is an isormorphism between holomorphic bundles $\left.\mathcal{E}_{0}\right|_{U \backslash \Sigma_{\infty}}$ and $\left.\mathcal{E}_{1}\right|_{U \backslash \Sigma_{\infty}}$. By the above discussion, it tends to $g_{\infty}$ as $z$ goes to 0 , so is bounded, hence extends holomorphically over $U \cap \Sigma_{\infty}$. Consequently, the bundle isomorphism $g$ extends over $U \cap \Sigma_{\infty}$ to give a bundle isomorphism of $\left.\mathcal{E}_{0}\right|_{U}$ and $\left.\mathcal{E}_{1}\right|_{U}$. The extended isomorphism is clearly unitary on $U \cap \Sigma_{\infty}$. We are done. 
Lemma 6.5 implies in particular that the extension of $E$ in Theorem 6.1 is independent of the choices of the various gauges and representatives of the flat limits on $Y$, for by Lemma 6.5 , the identity map of $E$ extends to an isomorphism between bundles constructed from different choices made in the construction. Thus these bundles are actually the same one under different holomorphic trivializations.

By Lemma 6.5, the class of $\mathcal{M}_{c u}^{*}(+,+)$ represented by the bundle $\mathcal{E}$ constructed in Theorem 6.1 depends only on the gauge equivalence class of the instanton $A$, thus there is a map from $\mathcal{M}^{*}(+,+)$ to $\mathcal{M}_{c u}^{*}(+,+)$, hence also $\mathcal{M}_{c}^{*}(+,+)$, which we define to be the map in Theorem 2.2.

To prove that the map is injective, it suffices to prove that the composite of it and the map defined in the last section is the identity map of $\mathcal{M}^{*}(+,+)$. But this follows trivially from Theorem 6.1 and Theorem 2.1. Thus the proof of Theorem 2.2 is completed.

In the rest of this section, we explain why if $\left(2^{\prime}\right)$ (see Sect. 2) is true, then the two maps constructed in the last section and this section would be inverses of each other.

So assume that $\left(2^{\prime}\right)$ is true, then the Hermitian metric $H$ of Theorem 2.1 satisfies (1) of the proposition in the appendix over both ends $\Sigma_{0}$ and $\Sigma_{\infty}$. By the proposition, $\left(3^{\prime}\right)$ also holds. Thus the map constructed in the last section is a map from $\mathcal{M}_{c}^{*}(+,+)$ to $\mathcal{M}^{*}(+,+)$ and is a left inverse of the map in Theorem 2.2 , and we only need to show that it is also a right inverse.

To this end, we start with a bundle $\mathcal{E}$ representing a given element of $\mathcal{M}_{c u}^{*}(+,+)$, apply Theorem 2.1, we get a Hermitian metric $H$ on $E$ whose Chern connection $A_{H}$ is an ASD connection on $(E, H)$ over $Y \times R$. The assumption that (2') holds implies that $A_{H}$ is an instanton on $(E, H)$. We then pull back $A_{H}$ to an instanton $A_{H_{0}}$ on $\left(E, H_{0}\right)$ by the gauge $g \in \operatorname{End}(E)$, positive self-adjoint with respect to both $H_{0}$ and $H$, determined by

$$
\langle\eta, \xi\rangle_{H_{0}}=\langle g \eta, g \xi\rangle_{H}
$$

for any sections of $E$. The connection $A_{H_{0}}$ then represents a class of $\mathcal{E}$ in $\mathcal{M}^{*}(+,+)$, which is the image of the map.

Now apply the other map to the element of $\mathcal{M}^{*}(+,+)$ represented by $A_{H_{0}}$, by definition we get the element of $\mathcal{M}_{c u}^{*}(+,+)$ represented by the bundle $\mathcal{E}_{0}$ constructed from $A_{H_{0}}$ in Theorem 6.1. On the other hand, it is clear that the bundle constructed from $A_{H}$ in Theorem 6.1 is the original bundle $\mathcal{E}$. Since $A_{H_{0}}$ and $A_{H}$ are gauge equivalent by $g \in \operatorname{End}(E)$, by Lemma $6.5 \mathcal{E}_{0}$ and $\mathcal{E}$ are isomorphic as bundles in $\mathcal{E}_{c}(+,+)$ by an isomorphism which extends $g$ and is unitary over the ends. Thus we come back to the original element of $\mathcal{M}_{c u}^{*}(+,+)$ represented by $\mathcal{E}$. So it indeed is also a right inverse.

\section{A. Appendix}

In this appendix, we prove Lemma 5.4 of Sect. 5 . We state the result in a slightly stronger way.

Proposition. Let $\mathcal{E} \in \mathcal{E}_{c}(+,+)$ be a holomorphic bundle over $S$ representing an element of $\mathcal{M}_{c}(+,+)$ and let $H_{0}^{\prime}$ be a Hermitian metric on $\mathcal{E}$ as in Lemma 5.2. Suppose $H$ is a Hermitian metric on $\left.\mathcal{E}\right|_{Y \times R}$ which is a bounded solution to the 
Hermitian Yang-Mills equation on $Y \times R$ with respect to $\Omega_{0}$. Then the following two conditions are equivalent:

(1) $H$ extends continuously over $\Sigma_{\infty}\left(\Sigma_{0}\right)$ on which it differs from $H_{0}^{\prime}$ by an endomorphism constant under flat unitary gauges.

(2) The following estimates hold:

$$
\begin{array}{rlrl}
\left|A_{H_{0}^{\prime}}-A_{H}\right| & =o(1), & t \rightarrow \infty(\text { resp. }-\infty), \\
\left|d_{A_{H_{0}^{\prime}}}\left(A_{H_{0}^{\prime}}-A_{H}\right)\right| & =o(1), \quad t \rightarrow \infty(\text { resp. }-\infty) .
\end{array}
$$

Proof. $(1) \Longrightarrow(2)$. Since the proof does not depend on which end is considered, we take the end to be $Y \times(0, \infty)$.

To start, let $B^{3}(0, r)$ be the ball of radius $r>0$ in $R^{3}$ centered at origin and $g=g_{l m} d x^{l} \otimes d x^{m}$, where $x$ is the standard coordinate of $R^{3}$, and let be a Riemannian metric on $B^{3}(0, r)$ such that

$$
g_{l m}=\delta_{l m}+O\left(|x|^{2}\right) .
$$

Consider the "box" $B^{3}(0, r) \times(-1,1)$ in $R^{3} \times R^{1}=R^{4}$ with the Riemannian metric $g+d t \otimes d t$ and the operator

$$
P_{g}=\frac{1}{2} \Delta_{g}+(\lambda-1) \frac{\partial}{\partial t}=\frac{1}{2} \Delta_{4}+\delta \Delta_{g}+(\lambda-1) \frac{\partial}{\partial t} .
$$

Since $\Delta_{g}=-\frac{1}{\sqrt{\operatorname{det} g}} \partial_{j}\left(g^{j k} \sqrt{\operatorname{det} g} \partial_{k}\right)$, it is easy to see that the error term $\delta \Delta_{g}$ is small in the sense that the coefficients of its second order entries are bounded by $O(|x|)$ and its first order by $O(1)$.

Let $D_{1} \supset D_{2} \supset D_{3}$ be relatively compact domains of $B^{3}(0, r) \times(-1,1)$. We need the following two standard elliptic estimates. One can find their proof in [11] from which we also adopt some notation here.

Lemma 1. Let $r$ be small enough. Let $0<\alpha<1$. For any $C^{2}$ function $u$ on $B^{3}(0, r) \times(-1,1)$,

$$
|u|_{1, \alpha ; \bar{D}_{2}} \leqq C\left(|u|_{0 ; \bar{D}_{1}}+\left|P_{g} u\right|_{0 ; \bar{D}_{1}}\right)
$$

where $C$ is a constant depending only on the bound of the error term $\delta \Delta_{g}$ and the domains $D_{1}$ and $D_{2}$.

Lemma 2. Let $r, \alpha$ be as in Lemma 1. For any $C^{2, \alpha}$ function $u$ on $B^{3}(0, r) \times$ $(-1,1)$,

$$
|u|_{2,0 ; \bar{D}_{3}} \leqq C\left(|u|_{0 ; \bar{D}_{2}}+\left|P_{g} u\right|_{0, \alpha ; \bar{D}_{2}}\right),
$$

where as above $C$ denotes a general constant depending only on the bound of the error term $\delta \Delta_{g}$ and the domains $D_{2}$ and $D_{3}$.

As in the main text, let $h$ be the automorphism of $E$ defined by $H=h^{t} H_{0}^{\prime}$. Then

$$
A_{H}-A_{H_{0}^{\prime}}=h^{-1} A_{H_{0}^{\prime}} h-A_{H_{0}^{\prime}}+h^{-1} \partial h
$$


and

$$
\begin{aligned}
& d_{A_{H_{0}^{\prime}}}\left(A_{H}-A_{H_{0}^{\prime}}\right)=d\left(A_{H}-A_{H_{0}^{\prime}}\right)+A_{H_{0}^{\prime}} \wedge\left(A_{H}-A_{H_{0}^{\prime}}\right)+\left(A_{H}-A_{H_{0}^{\prime}}\right) \wedge A_{H_{0}^{\prime}} \\
& =-h^{-1} d h \wedge h^{-1} A_{H_{0}^{\prime}} h+h^{-1} d A_{H_{0}^{\prime}} h-h^{-1} A_{H_{0}^{\prime}} \wedge d h-d A_{H_{0}^{\prime}}-h^{-1} d h h^{-1} \wedge \partial h \\
& \quad+h^{-1} \bar{\partial} \partial h+A_{H_{0}^{\prime}} \wedge\left(A_{H}-A_{H_{0}^{\prime}}\right)+\left(A_{H}-A_{H_{0}^{\prime}}\right) \wedge A_{H_{0}^{\prime}} .
\end{aligned}
$$

Let $e$ be a local holomorphic frame of $\mathcal{E}$ on a neighbourhood $U$ of some point at $\Sigma_{\infty}$ as in the remark following Lemma 5.2. We can cover the end by a finite number of such neighbourhoods. We then have, for the connection form $A_{H_{0}^{\prime}}$ under the frame $e$,

$$
\begin{aligned}
\left|A_{H_{0}^{\prime}}\right|=O\left(e^{-t}\right), \quad t \longrightarrow \infty, \\
\left|\nabla\left(A_{H_{0}^{\prime}}\right)\right|=O\left(e^{-t}\right), \quad t \longrightarrow \infty,
\end{aligned}
$$

in addition to

$$
\begin{aligned}
\left|F_{H_{0}^{\prime}}\right| & =O\left(e^{-t}\right), & t \longrightarrow \infty \\
\left|\nabla\left(F_{H_{0}^{\prime}}\right)\right| & =O\left(e^{-t}\right), & t \longrightarrow \infty .
\end{aligned}
$$

It suffices to show that

$$
|d h|=o(1), \quad t \longrightarrow \infty,
$$

and

$$
|\nabla(d h)|=o(1), \quad t \longrightarrow \infty,
$$

where $h$, by an absue of notation, is the matrix of the automorphism $h$ under the frame $e$ and $\nabla$ is the covariant derivative on forms by the Levi-Civita connection of $Y \times R$. We do this by refining a scaling argument initially used by Donaldson [6] on compact manifolds. Note that (1) implies that $h$ is a constant matrix, $h_{\infty}$ say, on $\Sigma_{\infty}$, and

$$
\left|h-h_{\infty}\right|=o(1), \quad t \longrightarrow \infty \text {. }
$$

To show

$$
|d h|=o(1), \quad t \longrightarrow \infty,
$$

we argue that the existence of a sequence of points $\left\{z_{j}=\left(y_{j}, t_{j}\right), t_{j} \rightarrow \infty\right\} \in Y \times R$ such that

$$
|d h|\left(z_{j}\right)=m_{j} \geqq c, \quad j \longrightarrow \infty
$$

for any given $c>0$ would lead to a contradiction. Choose a normal coordinate $\Omega_{j}$ of $y_{j}$ so that $\Omega_{j}$ is modeled on $B^{3}\left(0, r_{j}\right)$ with $y_{j}$ at the center and the Riemannian metric of $Y$ in this coordinate is given by

$$
g=\left(\delta_{l m}+C_{j}|x|^{2}\right) d x^{j} \otimes d x^{m}
$$

where $x$ is the standard coordinate in $B^{3}\left(0, r_{j}\right)$. Since $Y$ is compact, we can choose $r_{j}$ so that $r_{j} \geqq r$ if $r$ was chosen small and $C_{j}$ so that $C_{j} \leqq C$ for some $C$. Denote by $B_{j}^{4}$ the 4-dimensional ball of radius $\frac{r c}{m_{j}}$ and with center $\left(0, t_{j}\right)$ in $B^{3}\left(0, r_{j}\right) \times$ $\left(t_{j}-1, t_{j}+1\right)$. By taking another sequence if necessary, we may always assume that

$$
|d h|\left(z_{j}\right) \geqq \frac{1}{2} \sup _{z \in B_{j}^{4}}\{|d h|(z)\} .
$$


Now rescale each four ball $B_{j}^{4}$ to the standard four ball $B^{4}(0, r)$ in $B^{3}(0, r) \times$ $(-1,1)$. The Riemannian metric of $Y \times R$ in $B_{j}^{4}$ is pushed to

$$
g_{j}=\frac{c^{2}}{m_{j}^{2}}\left[\left(\delta_{l m}+\frac{c^{2}}{m_{j}^{2}} C_{j}|x|^{2}\right) d x^{l} \otimes d x^{m}+d t \otimes d t\right]
$$

on $B^{4}(0, r)$. Thus the operator $P$ on $Y \times R$ restricted to $B_{j}^{4}$ is equivalent to

$$
P_{g_{j}}=\frac{1}{2} \Delta_{g_{j}}+(\lambda-1) \frac{\partial}{\partial t}
$$

on $B^{4}(0, r)$. We shall mainly need their conformals

$$
g_{j}^{\prime}=\left(\delta_{l m}+\frac{c^{2}}{m_{j}^{2}} C_{j}|x|^{2}\right) d x^{l} \otimes d x^{m}+d t \otimes d t
$$

and

$$
P_{g_{j}^{\prime}}=\frac{1}{2} \Delta_{g_{j}^{\prime}}+(\lambda-1) \frac{\partial}{\partial t}=\frac{1}{2} \Delta_{4}+\delta \Delta_{g_{j}^{\prime}}+(\lambda-1) \frac{\partial}{\partial t} .
$$

Since the term $\frac{c^{2}}{m_{j}^{2}} C_{j}|x|^{2}$ in $g_{j}^{\prime}$ is uniformly bounded by $C|x|^{2}$, we see that the operator $\delta \Delta_{g_{j}^{\prime}}$ is unifomly small in an obvious sense.

Consider now the sequence of matrix functions $\left\{h_{j}\right\}$ on $B^{3}(0, r) \times(-1,1)$, where $h_{j}$ is the pullback of $h_{\Omega_{j} \times\left(t_{j}-1, t_{j}+1\right)}$. Clearly $\left|d h_{j}(o)\right|=c$ and $\left|d h_{j}(z)\right| \leqq 2\left|d h_{j}(0)\right|=$ $2 c$ with the metric $g_{j}^{\prime}$, hence also with the standard metric (replacing $c$ by some other constant if necessary). Note that the complex structure on $B_{j}^{4}$ induces a complex structure $\bar{\partial}_{j}$, say, on $B^{4}(0, r)$. For different $j, \bar{\partial}_{j}$ are in general different and in particular they are in general different from the standard complex structure on $B^{4}$, although in the case that the metric on $Y \times R$ is Kaehler we can choose the coordinates in $B_{j}^{4}$ so that $\bar{\partial}_{j}$ coincide with the standard one on $B^{4}(0, r)$ for all $j$. The metric $g_{j}^{\prime}$ is then a Hermitian metric with respect to the complex structure $\bar{\partial}_{j}$, thus there is an associated fundamental 2-form. Let $\Lambda_{j}^{\prime}$ be the adjoint to the wedge product by this fundamental 2-form on forms. The Hermitian Yang-Mills equation for $H$ on $Y \times R$ implies that, on $B^{4}(0, r)$,

$$
P_{g_{j}^{\prime}} h_{j}=i \Lambda_{j}^{\prime} \bar{\partial}_{j} h_{j} \wedge h_{j}^{-1} \partial_{j} h_{j}+i \Lambda_{j}^{\prime} \bar{\partial}_{j} h_{j} \wedge h_{j}^{-1} A_{H_{0}^{\prime}} h_{j}+i \Lambda_{j}^{\prime} A_{H_{0}^{\prime}} \wedge \bar{\partial}_{j} h_{j}-i \Lambda_{j}^{\prime} F_{H_{0}^{\prime}} h_{j} .
$$

The terms $\left|\partial_{j} h_{j}\right|$ and $\left|\bar{\partial}_{j} h_{j}\right|$ are bounded by $\left|d h_{j}\right| \leqq 2 c$ which does not depend on the complex structure.

By Lemma 1,

$$
\left|h_{j}-h_{\infty}\right|_{1, \alpha ; \bar{B}^{4}\left(0, \frac{2}{3} r\right)} \leqq C\left(\left|h_{j}-h_{\infty}\right|_{0 ; \bar{B}^{4}\left(0, \frac{3}{4} r\right)}+\left|P_{g_{j}^{\prime}} h_{j}\right|_{0 ; \bar{B}^{4}\left(0, \frac{3}{4} r\right)}\right) .
$$

We know that $\left|h_{j}-h_{\infty}\right|_{0 ; B^{4}(0, r)}=\left|\left(\left.h\right|_{B_{j}^{4}}-h_{\infty}\right)\right|=o(1)$, and since $\left|d h_{j}(z)\right| \leqq 2 c$ on $B^{4}(0, r)$, it is simple to see that the right-hand side of the preceding equation is bounded uniformly in $j$. Thus $\left|P_{g_{j}^{\prime}} h_{j}\right|_{0 ; B^{4}(0, r)}=\left|2 i \Lambda^{\prime} \bar{\partial}_{j} \partial_{j} h_{j}\right|_{0 ; B^{4}(0, r)}$ is also bounded. It follows from the above estimate that $h_{j}$ is bounded in $C^{1, \alpha}$ over $\bar{B}^{4}\left(0, \frac{2}{3} r\right)$. By 
Arzela's theorem we therefore conclude that, by going to a subsequence if necessary, $h_{j}$ converges in $C^{1}$ to some $h_{\infty}^{\prime}$ on $B^{4}\left(0, \frac{2}{3} r\right)$. In particular

$$
\left|d h_{\infty}^{\prime}(0)\right|=\lim _{j \rightarrow \infty}\left|d h_{j}(0)\right|=c .
$$

On the other hand, the points of the ball $B_{j}^{4}$ tend to infinity, thus $h_{j}$ tends uniformly to $h_{\infty}$, so $h_{\infty}^{\prime}=h_{\infty}$ which is constant under $e$. This contradicts $\left|d h_{\infty}^{\prime}(0)\right|=$ $c$. This shows

$$
|d h|=o(1), \quad r \longrightarrow \infty .
$$

To show

$$
|\nabla(d h)|=o(1), \quad t \longrightarrow \infty .
$$

Let $z$ be any point $(y, t) \in Y \times R$ "near" the end $\Sigma_{\infty}$. So it is in some $U$ as above. Choose a normal coordinate neighbourhood of $z$ modeled on $B(0, r)$ as above, but without scaling. Then as before the equation

$$
i \Lambda \bar{\partial} \partial h=i \Lambda \bar{\partial} h \wedge h^{-1} \partial h+i \Lambda \bar{\partial} h \wedge h^{-1} A_{H_{0}^{\prime}} h+i \Lambda A_{H_{0}} \wedge \bar{\partial} h-i \Lambda F_{H_{0}^{\prime}} h
$$

holds on $B(0, r)$ with the induced tube metric. By Lemma 2 ,

$$
|\nabla(d h)|_{0 ; \bar{B}\left(0, \frac{1}{2} r\right)} \leqq C\left(\left|h-h_{\infty}\right|_{0 ; \bar{B}\left(0, \frac{2}{3}\right) r}+|P h|_{0, \alpha ; \bar{B}\left(0, \frac{2}{3} r\right)}\right) .
$$

We know that $\left|h-h_{\infty}\right|_{0 ; \bar{B}\left(0, \frac{2}{3} r\right)}=o(1)$ and $|P h|_{0, \bar{B}\left(0, \frac{2}{3} r\right)}=o(1)$. So we only need to show that $|P h|_{\alpha ; \bar{B}\left(0, \frac{2}{3} r\right)}=o(1)$.

By Lemma 1 again, we have

$$
|h|_{1, \alpha ; \bar{B}\left(0, \frac{2}{3} r\right)} \leqq C\left(\left|h-h_{\infty}\right|_{0 ; \bar{B}\left(0, \frac{3}{4} r\right)}+\left|P_{g} h\right|_{0, \bar{B}\left(0, \frac{3}{4} r\right)}\right) .
$$

In particular

$$
|d h|_{\alpha ; \bar{B}\left(0, \frac{2}{3} r\right)}=o(1) .
$$

We also have

$$
\begin{gathered}
|h|_{\alpha ; \bar{B}\left(0, \frac{2}{3} r\right)} \leqq C|d h|_{0 ; B(0, r)}=o(1), \\
\left|A_{H_{0}^{\prime}}\right|_{\alpha ; \bar{B}\left(0, \frac{2}{3} r\right)} \leqq C\left|\nabla\left(A_{H_{0}^{\prime}}\right)\right|_{0 ; B(0, r)}=o(1),
\end{gathered}
$$

and

$$
\left|F_{H_{0}^{\prime}}\right|_{\alpha ; \bar{B}\left(0, \frac{2}{3} r\right)} \leqq C\left|\nabla\left(F_{H_{0}^{\prime}}\right)\right|_{0 ; B(0, r)}=o(1) .
$$

These and the general fact that

$$
|f g|_{\alpha ; \bar{B}\left(0, \frac{2}{3}\right)} \leqq|f|_{0 ; \bar{B}\left(0, \frac{2}{3}\right)}|g|_{\alpha ; \bar{B}\left(0, \frac{2}{3}\right)}+|f|_{\alpha ; \bar{B}\left(0, \frac{2}{3}\right)}|g|_{0 ; \bar{B}\left(0, \frac{2}{3}\right)}
$$

together imply that the $|\cdot|_{\alpha ; \bar{B}\left(0, \frac{2}{3} r\right)}$ norm of the right-hand side of the last equation above, hence also $|P h|_{\alpha ; \bar{B}\left(0, \frac{2}{3} r\right)}$ is bounded by $o(1)$. This completes the proof of the first step.

$(2) \Rightarrow(1)$. Let $h, e$ and $U$ be as above. It suffices to show that for every such local neighbourhood $U$ of $\Sigma_{\infty}$, the matrix form, denoted by $h$ itself, of the automorphism $h$ under the local frame $e$ extends over $\Sigma_{\infty}$ and is constant over there, for then we can define $H$ over $\Sigma_{\infty}$ by the formula $H=h^{t} H_{0}^{\prime}$ and by the boundedness of 
$H$ the extension must also be a Hermitian metric and also determine the given flat structure of $\left.\mathcal{E}\right|_{\Sigma_{\infty}}$.

To this end, let $\{z, w\}$ be local complex coordinates in $U$ as in Lemma 6.2. We can write

$$
d h=\frac{\partial h}{\partial z} d z+\frac{\partial h}{\partial \bar{z}} d \bar{z}+\frac{\partial h}{\partial w} d w+\frac{\partial h}{\partial \bar{w}} d \bar{w} .
$$

The condition

$$
\left|A_{H_{0}^{\prime}}-A_{H}\right|=o(1), \quad t \rightarrow \infty
$$

implies that

$$
|d h|=o(1), \quad t \rightarrow \infty
$$

By claim B) of Lemma 6.2,

$$
\text { (I) }\left|\frac{\partial h}{\partial z}\right|,\left|\frac{\partial h}{\partial \bar{z}}\right|=o\left(|z|^{-1}\right), \quad|z| \rightarrow 0
$$

and

$$
\text { (II) }\left|\frac{\partial h}{\partial w}\right|,\left|\frac{\partial h}{\partial \bar{w}}\right|=o(1), \quad|z| \rightarrow 0 .
$$

It follows from (I) that $h(z, w)$ converges to some $h(0, w)$ as $z \rightarrow 0$ and from (II) $h(0, w)$ is constant.

Acknowledgements. This paper is a slight variant of some part of my thesis. I am immensely grateful to Simon Donaldson for initially suggesting the topic, for continuous advice and encouragements throughout my Ph.D. years. I also wish to thank Peter Kronheimer and Dietmar Salamon for encouragements and for pointing out many mistakes. Most of the mistakes were corrected during my stay at the Max-Planck Institut and I thank the Institut for its hospitality and financial support. Finally, I am grateful to the referee whose numerous suggestions certainly improve the readability of this paper.

\section{References}

1. Atiyah, M.F., Patodi, V.K., Singer, I.M.: Spectral asymmetry and Riemannian Geometry. I. Math. Proc. Cambridge Philos. Soc. 77, 43-69 (1975); II, Math. Proc. Cambridge Philos. Soc. 78, 405-432 (1975); III, Math. Proc. Cambridge Philos. Soc. 79, 71-99 (1976)

2. Bando, S.: Einstein-Hermitian metrics on non-compact Kaehler manifols. Max-Planck-Institut, Preprint

3. Biquard, O.: Fibre Paraboliques Stables et Connexions Singulieres Plates. Bull. Soc. Math. France 119, 231-257 (1991)

4. Buchdahl, N.P.: Hermitian-Einstein Connections and Stable Vector Bundles Over Compact Complex Surfaces. Math. Ann. 280, 625-648 (1998)

5. Chavel, I.: Eigenvalues in Riemannian geometry. New York: Academic Press, 1984

6. Donaldson, S.K.: Anti self-dual Yang-Mills connections over complex algebraic surfaces and stable vector bundles. Proc. London Math. Soc. 50, 1-26 (1985)

7. Donaldson, S.K.: Boundary value problems for Yang-Mills fields. J. Geom. and Phys. 8, 89$122(1992)$

8. Donaldson, S.K., Furuta, M., Kotshick, D.: Floer homology groups in Yang-Mills theory. In preparation

9. Fukaya, K.: Floer homology for oriented homology 3-spheres. Advanced Studies in Pure Mathematics 20, Tokyo, Kinokuniya Company Ltd., 1992, pp. 1-92

10. Furuta, M., Steer, B.: Seifert Fibered Homology 3-spheres and the Yang-Mills Equations. Advances in Mathematics 96, No. 1, 38-102 (1992) 
11. Gilbarg, D., Trudinger, N.S.: Elliptic partial differential equations of second order. Second edition, Berlin and New York: Springer-Verlag, 1983

12. Guo, G.-Y.: Thesis, Oxford

13. Hamilton, R.S.: Harmonic maps of manifolds with boundary. LNM 471, Berlin: Springer, 1982

14. Hamilton, R.S.: Three manifolds with positive Ricci curvature. J. Diff. Geom. 17, 253-306 (1982)

15. Jost, J.: Nonlinear methods in Riemannian and Kaehlerian Geometry. DMV Seminar Band 10, Basel-Boston: Birkhauser Verlag, 1988

16. Kobayashi, S.: Differential geometry of complex vector bundles. Publ. Math. Soc. Japan, Iwanami Shoten and Princeton Univ., 1987

17. Kroheimer, P., Mrowka, T.: Gauge theory for embedded surfaces, I. Topology 32, No. 4, 773-826 (1993); II, Topology 34, 37-97 (1995)

18. Narasimhan, M.S., Seshadri, C.S.: Stable and unitary bundles on a compact Riemann surface. Ann. Math. 82, 540-564 (1965)

19. Simpson, C.T.: Constructing variations of Hodge structure using Yang-Mills theory and applications to uniformization. J. Am. Math. Soc. 1, 867-918 (1988)

20. Taubes, C.H.: $L^{2}$-moduli spaces on 4-manifolds with cylindrical ends. International Press, Hong Kong, 1994

21. Uhlenbeck, K.: Connections with $L^{p}$ bounds on curvature. Commun. Math. Phys. 83, 31-42 (1982)

22. Uhlenbeck, K., Yau, S.T.: On the existence of Hermitian-Yang-Mills connections in stable vector bundles. Commun. Pure and Appl. Math. 39-S, 257-293 (1986)

Communicated by S.-T. Yau 
